\title{
PHYTOCHEMICALS AS POTENTIAL INHIBITORS OF LANOSTEROL 14 A-DEMETHYLASE (CYP51) ENZYME: AN IN SILICO STUDY ON SIXTY MOLECULES
}

\author{
ASHWINI KHANDERAO JADHAV ${ }^{1}$, PATHAN KAMRAN KHAN², SANKUNNY MOHAN KARUPPAYIL ${ }^{*}$
}

${ }^{1}$ Department of Stem Cell and Regenerative Medicine, Centre for Interdisciplinary Research, DY Patil Education Society (Deemed to be University, NAAC Accredited with 'A' Grade), Kolhapur, Maharashtra, India 416006. ${ }^{2}$ School of Life Sciences (DST-FIST and UGC-SAP Sponsored), SRTM University (NAAC Accredited with ' $A$ ' grade), Nanded, Maharashtra State, India, 431606. ${ }^{*}$ Department of Stem Cell and Regenerative Medicine, Centre For Interdisciplinary Research, DY Patil Education Society (Deemed to be University, NAAC Accredited with 'A' Grade), Kolhapur, Maharashtra, India 416006

Email: prof.karuppayil@gmail.com

Received: 3 Jun 2020, Revised and Accepted: 15 Jul 2020

\section{ABSTRACT}

Lanosterol $14 \alpha$-demethylase (CYP51) is a key protein involved in ergosterol biosynthesis of Candida albicans and a crucial target for ergosterol synthesis inhibition. However, in the last two decades drug resistance is reported under clinical situations to most of the prescribed antifungal drugs like azole group of drugs. In this study, molecular docking of sixty plant molecules with Lanosterol $14 \alpha$-demethylase protein has been done. The homology modeling tool PHYRE2 was used to predict the structure of Lanosterol $14 \alpha$-demethylase. Predicted structure was used for docking studies with sixty plant molecules by using Autodock $1.5 .6 \mathrm{cr} 2^{\mathrm{TM}}$. Among the sixty plant molecules, forty-seven were found to form hydrogen bond and the rest of the plant molecules did not form a hydrogen bond with Lanosterol $14 \alpha$-demethylase. Docking study of a library of sixty molecules revealed that 48 plant molecules showed an excellent and good binding affinity with predicted protein model Lanosterol $14 \alpha$-demethylase of Candida albicans. The binding residue comparison of docked molecules with that of Ketoconazole revealed, fourteen molecules have similar binding residue. These fourteen molecules may have a similar mode of action as that of Ketoconazole. These molecules should be screened and used to discover new antifungal therapeutic drugs.

Keywords: Lanosterol $14 \alpha$-demethylase, Phytochemicals, Molecular docking, Candida albicans, Ergosterol synthesis

(C) 2020 The Authors. Published by Innovare Academic Sciences Pvt Ltd. This is an open access article under the CC BY license (http://creativecommons.org/licenses/by/4.0/) DOI: http://dx.doi.org/10.22159/ijap.2020.v12s4.40100. Journal homepage: https://innovareacademics.in/journals/index.php/ijap

\section{INTRODUCTION}

The prevalence of opportunistic fungal infections has blown up in the couple of years [1]. 1.5 to 2 million deaths occur every year due to fungal infections in immunocompromised patients such as those suffering from autoimmune diseases, AIDS, burns and chemo or radiotherapy [2]. One of the most commonly used drugs for the prevention of Candidiasis is Fluconazole, a member of the azole family. Its target is an essential enzyme, Lanosterol $14 \alpha$-demethylase a member of the cytochrome P450 superfamily. This is a heme thiolate enzyme which converts lanosterol into 4,4'-dimethyl cholesta-8,14,24-triene-3-beta-ol [3]. The activity of azole drugs is attributed to the co-ordinate binding of the heterocyclic nitrogen atom (N-3 of imidazole and N-4 of triazole) to the heme iron atom in the binding site of CYP51 enzyme. Inhibition of CYP51, and depletion of ergosterol coupled with the accumulation of 14methyl sterols results in impaired fungal growth [4]. The vital role of CYP51 in fungal metabolism makes it an ideal target for antifungal drug design [5]. Numerous classes of the drugs have been developed which target the ergosterol biosynthetic pathway [6, 7].

To treat fungal infection, there are five classes of drugs. These are Polyenes, azoles, echinocandins, allylamines and fluoropyrimidines. In addition to drug resistance, acute and chronic side effects, less clinical efficiency and effect on non-target cells are the hitch of the existing drugs and therefore, researchers around the world are in the search for novel and efficient antifungal drugs [8]. Resistance towards the drugs and side effects clearly indicates that there is a need for development of new drugs. Researchers has previously indicated that the structurally and functionally essential regions, such as the heme group, the hydrophilic H-bonding region, the narrow hydrophobic cleft-substrate access channel 2 (FG loop), and the active site could be good targets for antifungal drugs. The binding mode of azoles with lanosterol $14 \alpha$-demethylase protein of Candida albicans CYP51 has been investigated through molecular docking $[9,10]$. The molecular modeling can accelerate the discovery of novel antifungal agents through the exploitation of structural in order of fungal CYP51s [11].

In the present work, we have screened a library of sixty molecules for molecular docking with the predicted structure of lanosterol
$14 \alpha$-demethylase protein of Candida albicans to investigate their binding affinity in search of Phytochemicals as potent antifungal drugs.

\section{MATERIALS AND METHODS}

Homology modeling of Lanosterol $14 \alpha$-demethylase (CYP51)

Primary sequence of Lanosterol $14 \alpha$-demethylase (CYP51) was retrieved in FASTA format from the Uniprot public domain protein database (Uniprot accession no. P10613). Retrieved sequence was submitted to the Phyre2 homology modeling program for modeling of the three-dimensional structure of the protein [12]. Tertiary structure was predicted and Validation of tertiary structure was done by Procheck [13]. Tertiary structure of Lanosterol $14 \alpha-$ demethylase (CYP51) was used for docking studies [14].

\section{Protein structure preparation}

The Autodock Tools package version 1.5.6 rc 2 was employed to generate the docking input files.

All the nonpolar hydrogens were merged and the water molecules were removed. For Docking, a grid spacing of $0.375 \AA$ and $60 \times 60 \times 60$ number of points was used. Before docking all water molecules were removed from the protein structure, followed by addition of Hydrogen atoms to receptor and merging non-polar hydrogens. Modeled three dimensional structure of Lanosterol $14 \alpha$-demethylase and the structure of each ligand were converted to PDBQT format [14, 15].

\section{Ligand structure preparation}

The structures of all the molecules were retrieved from Pubchem, chemical structure followed by 2D structure cleaning, 3D optimization and viewing. Molecular docking study of molecules against Lanosterol 14 $\alpha$-demethylase was carried out. Docking simulation was done using AutoDock ${ }^{\circledR}$ suite as a molecular-docking tool [15]. Default optimization parameters were used Lamarckian Genetic Algorithm was used with a population size of 150 dockings. Autodock ${ }^{\circledR}$ tools generated 60 possible binding conformations, i.e. 60 runs for each docking by using Genetic Algorithm (GALS) searches. The grid box used for specifying the search space was set at $60 \times 60 \times 60$ centered on of Protein with a default grid point spacing of $0.375 \AA$ Å. Autogride was used to obtain pre-calculated 
grid maps. 25.84083, 10.02083 and 9.119833 were used as $\mathrm{x}, \mathrm{y}$ and $\mathrm{z}$ coordinate during Grid preparation. Docking of molecules with the predicted structure on Lanosterol 1,4 $\alpha$-demethylase was done. After completion of docking, most suitable conformations were chosen based on the lowest docked energy. Selected conformations were analyzed by Autodock $^{\circledR}$ tool and Discovery studio ${ }^{\circledR}[14,15]$.

Table 1: Interaction of molecules with Candida albicans lanosterol 1,4 $\alpha$-demethylase (CYP51)

\begin{tabular}{|c|c|c|c|c|c|c|c|}
\hline Groups & S. No. & Molecules & $\begin{array}{l}\text { Interacting residue } \\
\text { in alpha- } \\
\text { demethylase }\end{array}$ & $\begin{array}{l}\text { Interacting atoms } \\
\text { (amino acid.... } \\
\text { Ligand) }\end{array}$ & $\begin{array}{l}\text { H- } \\
\text { bonds } \\
\text { formed }\end{array}$ & $\begin{array}{l}\text { Binding } \\
\text { Energy } \\
\text { (Kcal/mol) } \\
\end{array}$ & $\begin{array}{l}\text { Electrostatic } \\
\text { Energy }\end{array}$ \\
\hline \multirow[t]{23}{*}{ Excellent binding } & 1. & Ketoconazole & LYS143 & HZ3 .... N4 & 1 & -11.85 & -0.31 \\
\hline & 2. & Hesperidin & TYR118 & $\mathrm{OH} \ldots . \mathrm{H} 20$ & 5 & -9.76 & -0.2 \\
\hline & & & GLY307 & O.... H31 & & & \\
\hline & & & THR311 & HG1 .... 015 & & & \\
\hline & & & LYS143 & HZ3 .... 06 & & & \\
\hline & & & TYR132 & НH.... $\mathrm{O} 3$ & & & \\
\hline & 3. & Quinine & THR311 & $\mathrm{HG} 1 \ldots . . \mathrm{O} 2$ & 2 & -9.2 & -0.06 \\
\hline & & & ILE471 & HN.... 01 & & & \\
\hline & 4. & Riboflavin & ILE471 & HN.... 01 & 4 & -8.56 & -0.44 \\
\hline & & & HIS468 & $\mathrm{H} 20 \ldots . \mathrm{O}$ & & & \\
\hline & & & LYS143 & HZ3 .... O4 & & & \\
\hline & & & TYR132 & $\mathrm{OH} \ldots . \mathrm{H} 12$ & & & \\
\hline & 5. & Piperine & LYS143 & $\mathrm{OH} \ldots . . \mathrm{O} 3$ & 2 & -8.54 & -0.09 \\
\hline & & & TYR132 & $\mathrm{HZ3} \ldots . . \mathrm{O} 2$ & & & \\
\hline & 6. & Rutin-trihydrate & HIS468 & О.... H29 & 3 & -8.52 & -0.42 \\
\hline & & & TYR132 & НН.... 06 & & & \\
\hline & & & TYR132 & $\mathrm{OH} \ldots . \mathrm{H} 20$ & & & \\
\hline & 7. & Caryophyllene-oxide & LYS143 & $\mathrm{HZ3} \ldots . . \mathrm{O} 1$ & 1 & -7.66 & -0.28 \\
\hline & 8. & Quercetin & HIS468 & O.... H9 & 5 & -7.54 & -0.36 \\
\hline & & & GLY307 & O.... H8 & & & \\
\hline & & & THR311 & HG1 .... 05 & & & \\
\hline & & & LYS143 & HZ3 .... O6 & & & \\
\hline & & & TYR132 & $\mathrm{OH} \ldots . . \mathrm{H} 10$ & & & \\
\hline \multirow[t]{10}{*}{ Good } & 9. & Betaionone & LYS143 & HZ3 .... 01 & 1 & -6.95 & -0.39 \\
\hline & 10. & Alpha-bisabolol & ILE471 & HN.... 01 & 1 & -6.91 & -0.08 \\
\hline & 11. & Fluconazole & ARG469 & $\mathrm{O} \ldots . . \mathrm{H} 5$ & 2 & -6.82 & -0.33 \\
\hline & & & LYS143 & $\mathrm{HZ3} \ldots . . \mathrm{N} 4$ & & & \\
\hline & 12. & Indole-3-butyric-acid & LYS143 & $\mathrm{HZ3} \ldots . . \mathrm{O} 2$ & 1 & -6.73 & -0.34 \\
\hline & 13. & Geranylgeranoil & LYS143 & HZ3 .... 01 & 1 & -6.67 & -0.25 \\
\hline & 14. & Geranylacetate & LYS143 & $\mathrm{HZ3} \ldots . . .02,01$ & 1 & -6.08 & -0.36 \\
\hline & 15. & Farnesol & HIS468 & O.... H26 & 2 & -6.06 & -0.15 \\
\hline & & & LYS143 & HZ3 .... 01 & & & \\
\hline & 16. & Caffeine & ILE471 & HN.... O2 & 1 & -6.02 & -0.11 \\
\hline \multirow[t]{35}{*}{ Medium } & 17. & Caffeic-acid & GLY307 & $0 \ldots . \mathrm{H} 6$ & 3 & -5.79 & -0.24 \\
\hline & & & THR311 & $\mathrm{HN} \ldots . . \mathrm{O} 2$ & & & \\
\hline & & & MET306 & $\mathrm{O} \ldots . \mathrm{H} 7$ & & & \\
\hline & 18. & Citral & LYS143 & HZ3 .... 01 & 1 & -5.76 & -0.35 \\
\hline & 19. & Cinnamic-acid & LYS143 & HZ3 .... 02 & 1 & -5.75 & -0.43 \\
\hline & 20. & Carvacrol & GLN479 & OE1 .... H14 & 2 & -5.67 & -0.05 \\
\hline & & & GLN479 & HE21.... 01 & & & \\
\hline & 21. & Citronellol & LYS143 & HZ3 .... 01 & 1 & -5.48 & -0.32 \\
\hline & 22. & Geraniol & HIS468 & O.... H18 & 1 & -5.48 & -0.26 \\
\hline & 23. & Carvone & LYS143 & HZ3 .... 01 & 1 & -5.47 & -0.35 \\
\hline & 24. & 1-8,cineole & SER378 & 0.... H18 & 1 & -5.45 & -0.04 \\
\hline & 25. & Salicylic-acid & HIS468 & O.... H5 & 2 & -5.44 & -0.49 \\
\hline & & & LYS143 & $\mathrm{HZ3} \ldots . . \mathrm{O} 3$ & & & \\
\hline & 26. & Borneol & LYS143 & HZ3 .... 01 & 1 & -5.43 & -0.40 \\
\hline & 27. & Menthol & ILE471 & HN.... O1 & 1 & -5.38 & -0.03 \\
\hline & 28. & Eugenol & HIS468 & O.... H10 & 2 & -5.38 & -0.19 \\
\hline & & & LYS143 & $\mathrm{HZ3} \ldots . . \mathrm{O} 1$ & & & \\
\hline & 29. & Methyleugenol & HIS468 & $0 \ldots .02$ & 2 & -5.36 & -0.16 \\
\hline & & & LYS143 & HZ3 .... 01 & & & \\
\hline & 30. & Isopulegol & ILE304 & O.... H13 & 1 & -5.32 & -0.03 \\
\hline & 31. & 1-4, cineole & ILE304 & $0 \ldots . .01$ & 1 & -5.31 & -0.05 \\
\hline & 32. & Nerol & LYS143 & HZ3 .... 01 & 2 & -5.29 & -0.26 \\
\hline & & & HIS468 & О.... H18 & & & \\
\hline & 33. & Alpha-thujone & LYS143 & HZ3 .... 01 & 1 & -5.22 & -0.24 \\
\hline & 34. & Sabinene-hydrate & SER378 & О......H18 & 1 & -5.22 & -0.05 \\
\hline & 35. & Thymol & ILE304 & O.... H14 & 1 & -5.16 & -0.06 \\
\hline & 36. & Cinnamaldehyde & LYS143 & HZ3 .... 01 & 1 & -5.1 & -0.22 \\
\hline & 37. & Betacitronellol & HIS468 & O.... H14 & 1 & -5.03 & -0.14 \\
\hline & 38. & Nicotinic-acid & LYS143 & $\mathrm{HZ3} \ldots . . \mathrm{O} 2$ & 1 & -4.92 & -0.4 \\
\hline & 39. & Indole & HIS468 & O.... H1 & 1 & -4.81 & -0.06 \\
\hline & 40. & 1-tetradecanol & HIS468 & О.... Н30 & 2 & -4.77 & -0.38 \\
\hline & & & LYS143 & HZ3 .... 01 & & & \\
\hline & 41. & Ascorbic-acid & HIS468 & O.... H7 & 4 & -4.76 & -0.49 \\
\hline & & & TYR132 & OH.... H8 & & & \\
\hline & & & HIS468 & $0 \ldots \mathrm{H} 5$ & & & \\
\hline
\end{tabular}




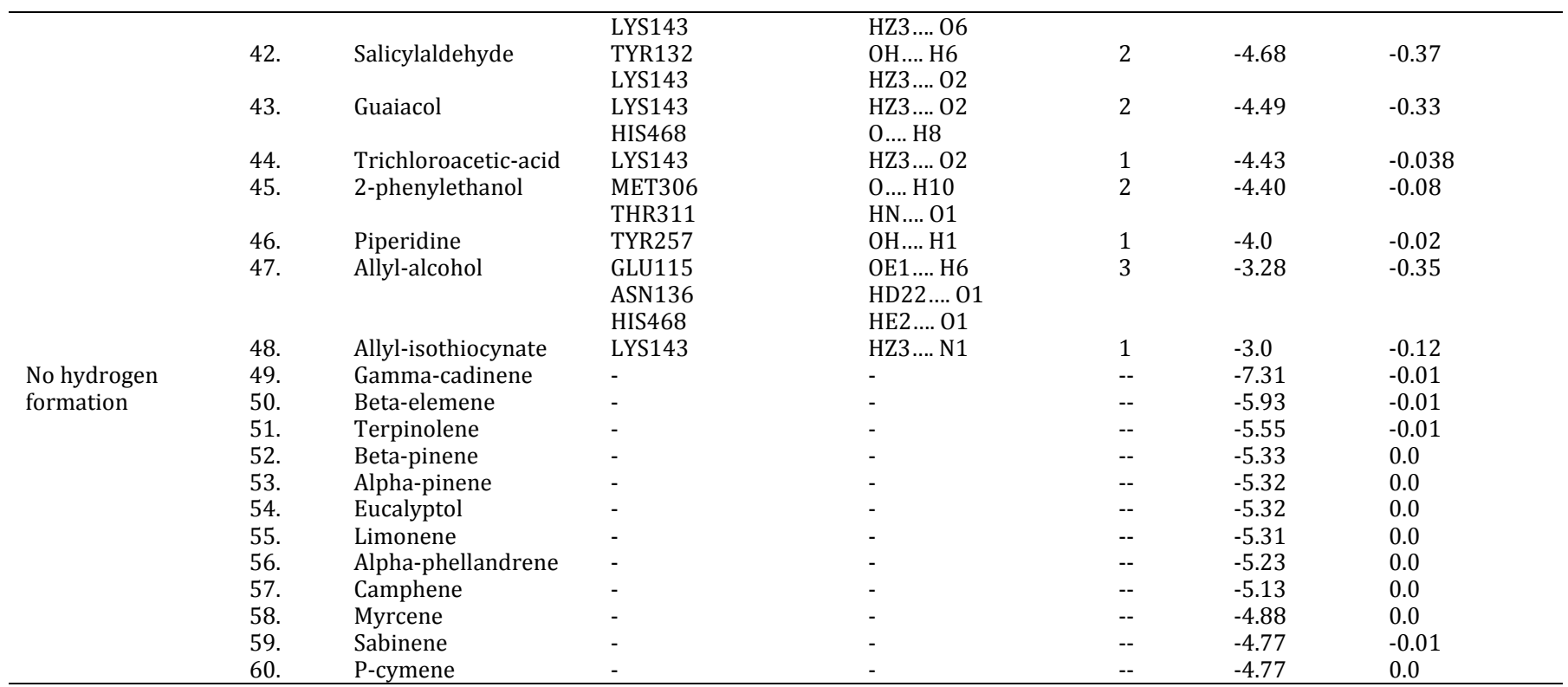

\section{RESULTS}

Homology modeling and molecular docking against lanosterol $14 \alpha$-demethylase

Molecular docking of sixty plant molecules with the best predicted model of Lanosterol $14 \alpha$-demethylase CYP51 protein was successfully done using Autodock $1.5 .6 \mathrm{cr} 2^{\mathrm{TM}}$. When validation was done by Procheck, it found that $90.2 \%$ residues are in most favored regions $[\mathrm{A}, \mathrm{B}, \mathrm{L}]$. Docking results of sixty plant molecules with Lanosterol $14 \alpha$-demethylase was calculated on the basis of RMSD values and compared with that of standard drugs (Fluconazole and Ketoconazole). The results of all the sixty docked molecules are listed in table 1 . Docked plant molecules showed binding energy with a range of- 9.76 to- $3.0 \mathrm{kcal} / \mathrm{mol}$. The lowest binding energy or more negative energy was considered to be the best docking results.

After docking 60 best runs having the lowest binding energy was chosen as best candidates for building a complex of Ligand and Lanosterol $14 \alpha$-demethylase protein. From the best-chosen candidates, seven plant molecules were shown to have excellent binding energy, namely Hesperidin, Quinine, Riboflavin, Piperine, Rutin-trihydrate, Caryophyllene-oxide and Quercetin (table 1) Hesperidin showed to minimum binding energy- $9.76 \mathrm{kcal} / \mathrm{mol}$ and formation of a hydrogen bond at TYR118, GLY307, THR311, LYS143 and TYR132 residues of protein (fig. 1). Quinine formed two hydrogen bonds at TYR132 AND ILE471 with binding energy 9.2 $\mathrm{Kcal} / \mathrm{mol}$ (fig. 2). Riboflavin showed the formation of four hydrogen bonds with ILE471, HIS468, LYS143 and TYR132 having binding energy-8.56 kcal/mol (fig. 3). Piperine was found to form two hydrogen bonds with LYS143 and TYR132 with the-8.54 kcal/mol binding energy (fig. 4). Rutin-trihydrate was observed to bind with HIS468, TYR132 and TYR132 by three hydrogen bonds with binding energy-8.52 kcal/mol (fig. 5). Caryophyllene-oxide formed one hydrogen bond LYS143 amino acid residue with binding energy-7.66 $\mathrm{kcal} / \mathrm{mol}$ (fig. 6). Quercetin formed five hydrogen bonds with HIS468, GLY307, THR311, LYS143 and TYR143, having binding energy-7.54 kcal/mol (fig. 7). Betaionone, Alpha-bisabolol, Geranylgeranoil, Indole-3-butyric acid and Geranylacetate showed the formation of one-one hydrogen bonds with amino acid residue LYS143, ILE143, ILE471, LYS143 and LYS143 with binding energy6.95,-6.91,-6.67,-6.73 and-6.08 kcal/mol respectively (fig. 8-12). Farnesol formed two hydrogen bonds with amino acid residues of$6.06 \mathrm{kcal} / \mathrm{mol}$ (fig. 13). Caffeine formed H-bond with ILE471 having binding energy-6.02 kcal/mol (fig. 14). Caffeic-acid formed three hydrogen-bonds with GLY307, THR311 and MET306 by binding energy-5.79 kcal/mol (fig. 15). Citral and Cinnamic acid showed a hydrogen bond with LYS143 and LYS143 with binding energy of5.76 and $-5.75 \mathrm{kcal} / \mathrm{mol}$ (fig. 16, 17). Carvacrol was found to form two hydrogen-bonds with GLN479 and GLN479 having binding energy-5.67 kcal/mol (fig. 18). Citronellol, Geraniol, Carvone and 1, 8 Cineole showed hydrogen bond with LYS143, HIS468, LYS143 and SER378 with binding energy-5.48,-5.48,-5.47 and-5.45 kcal/mol (fig. 19-22). Salicylic-acid was found to form two hydrogen bonds with HIS468 and LYS143 with binding energy of-5.44 kcal/mol (fig. 23) Borneol and Menthol were found to form one-one hydrogen bond with LYS143 and ILE471 amino acid residues with-5.43 and-5.38 $\mathrm{kcal} / \mathrm{mol}$ binding energy respectively (fig. 24-25). Eugenol was found to form two hydrogen bonds HIS468 and LYS143, with-5.38 kcal/mol binding energy (fig. 26). Methyleugenol was formed two hydrogen bonds with HIS468 and LYS143 having-5.38 kcal/mol (fig. 27). Isopulegol is shown to form hydrogen bond with amino acid residue ILE304 with binding energy of-5.36 kcal/mol (fig. 28). 1, 4 Cineole form hydrogen bond with ILE304 with-5.31kcal/mol binding energy (fig. 29). Nerol formed a two hydrogen bond LYS143 and HIS468 having-5.29 kcal/mol (fig. 30). Seven molecules namely Alpha-thujone, Sabinene-hydrate, Thymol, Cinnamaldehyde, Betacitronellol, Nicotinic-acid and Indole was found to form a one-one hydrogen bond with amino acid residues, LYS143, SER378, ILE304, LYS143, HIS468, LYS143, LYS143 and HIS468 respectively, with having binding energy of-5.22,-5.22,-5.16,-5.1,-5.03,-4.92 and $-4.81 \mathrm{kcal} / \mathrm{mol}$ (fig. 31-37). 1-tetradecanol found to form two hydrogen bonds HIS468 and LYS143 with amino acid residues having- $4.77 \mathrm{kcal} / \mathrm{mol}$ binding energy (fig. 38). Ascorbic-acid formed four hydrogen bond with HIS468, TYR132, HIS468, and LYS132 having-4.76 kcal/mol (fig. 39). Salicylaldehyde formed two hydrogen bonds TYR132 and LYS143 having-4.68 kcal/mol binding energy (fig. 40). Guaiacol formed two hydrogen bonds with LYS143 and HIS468 with binding energy of-4.49 kcal/mol (fig. 41). Trichloroacetic-acid forms a hydrogen bond with LYS143 amino acid residue with- $4.43 \mathrm{kcal} / \mathrm{mol}$ binding energy (fig. 42). 2-Phenylethanol formed two hydrogen bonds with MET306 and THR311 were having-4.40 kcal/mol binding energy (fig. 43). Piperidine was found to form hydrogen bond with TYR257 having-4.0 kcal/mol binding energy (fig. 44). Allyl-alcohol formed three hydrogen bonds GLU15, ASN136 and HIS468 having binding energy-3.28 kcal/mol (fig. 45). Allylisothiocynate formed one hydrogen bond with LYS143 with binding energy $3.0 \mathrm{kcal} / \mathrm{mol}$ (fig. 46)

Fluconazole and Ketoconazole were taken as standard drugs, which are widely used as antifungal agents. Fluconazole formed two hydrogen bonds ARG469 and LYS143, having-6.82 kcal/mol binding energy (fig. 47). Ketoconazole also formed hydrogen bond with LYS143 with amino acid residues having-11.85 $\mathrm{kcal} / \mathrm{mol}$ binding energy with protein (fig. 48).

However, Rest of the molecules did not form hydrogen bond with amino acid residues these molecules are. Alpha-pinene, Beta-pinene, Camphene, Beta-elemene, Alpha-phellandrene, Eucalyptol, Myrcene, P- 
cymene, Sabinene, Terpinolene, Gamma, cadinene, Limonene. Docking results of sixty plant molecules with Lanosterol $14 \alpha$-demethylase was compared to Ketoconazole on the basis of binding residue LYS143. The comparison shows that 14 molecules Caryophyllene-oxide, Betaionone,

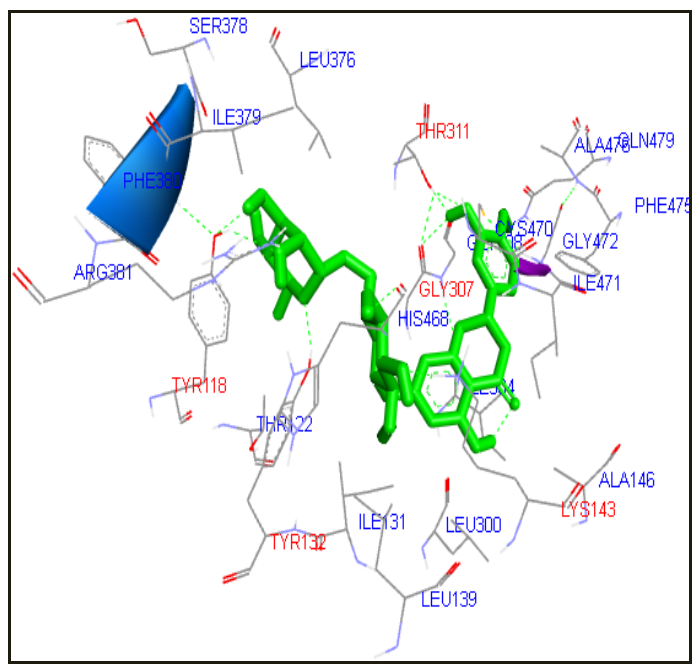

Fig. 1: Docked complex showing hesperidin with lanosterol $14 \alpha$ demethylase

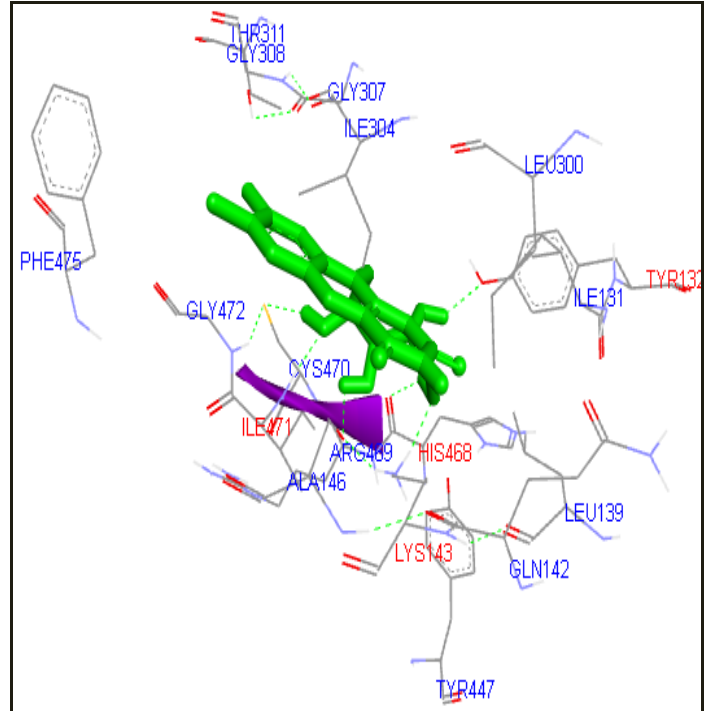

Fig. 3: Docked complex showing Riboflavin with lanosterol $14 \alpha$ demethylase

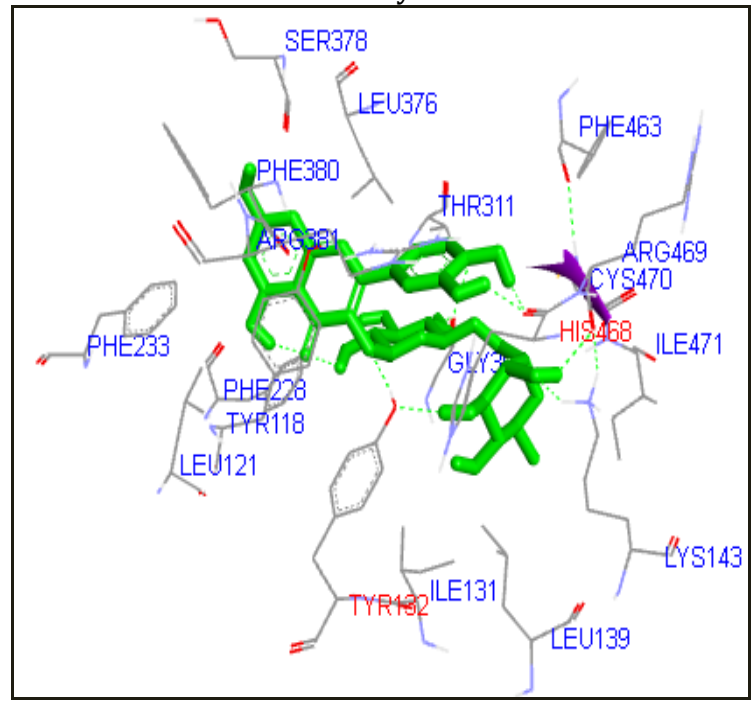

Fig. 5: Docked complex showing Rutin-trihydrate with lanosterol $14 \alpha$-demethylase
Indole-3-butyric-acid, Geranylgeranoil, Geranylacetate, Citral, Cinnamicacid, Citronellol, Carvone, Borneol, Alpha-thujone, Cinnamaldehyde, Nicotinic-acid, and Allyl-alcohol interacted with LYS143 of Lanosterol 14 $\alpha$-demethylase similar to that of Ketoconazole.

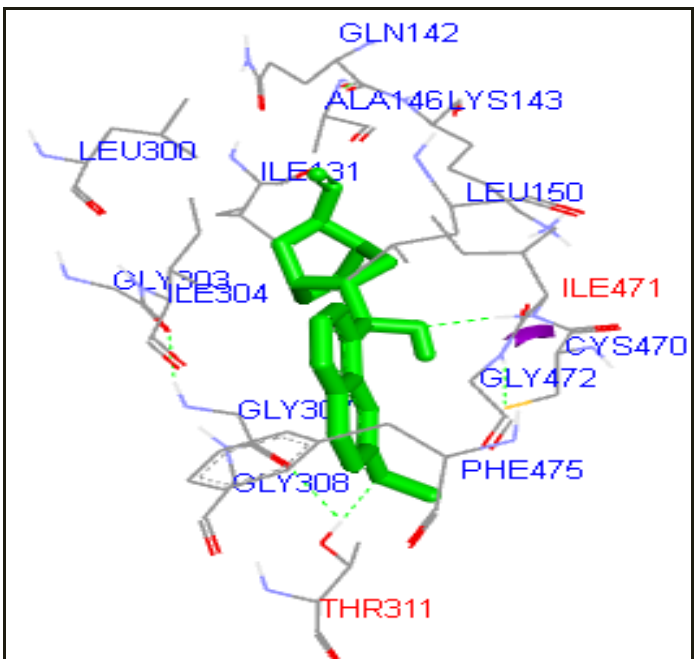

Fig. 2: Docked complex showing quinine with lanosterol $14 \alpha$ demethylase

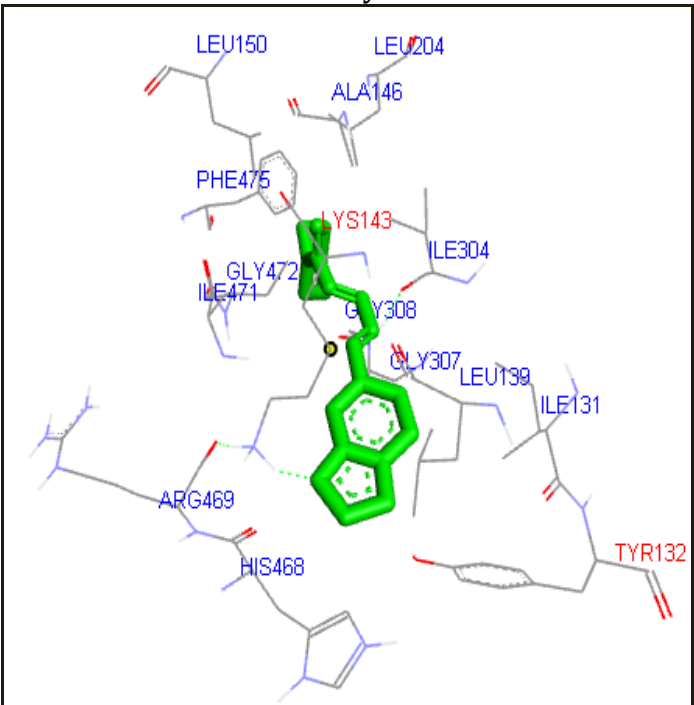

Fig. 4: Docked complex showing Piperine with lanosterol $14 \alpha$ demethylase

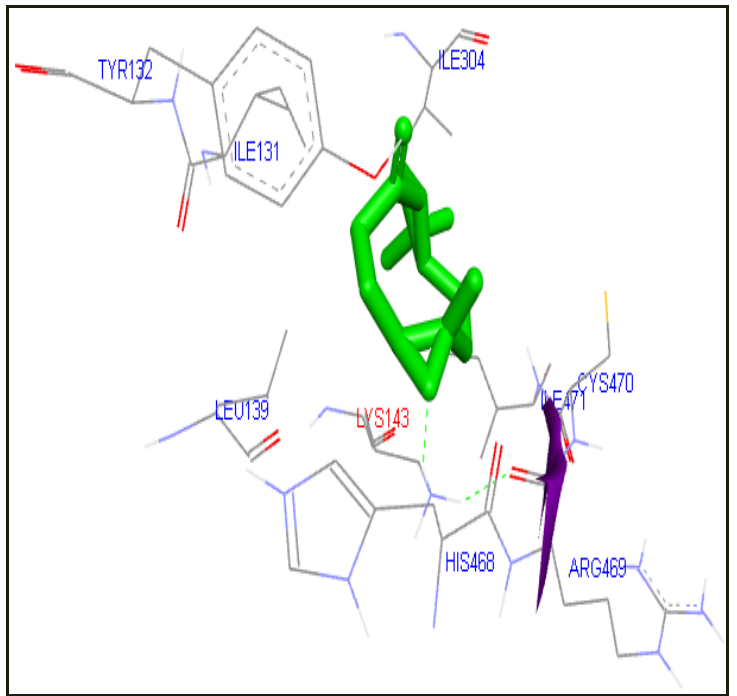

Fig. 6: Docked complex showing caryophyllene-oxide with lanosterol $14 \alpha$-demethylase 


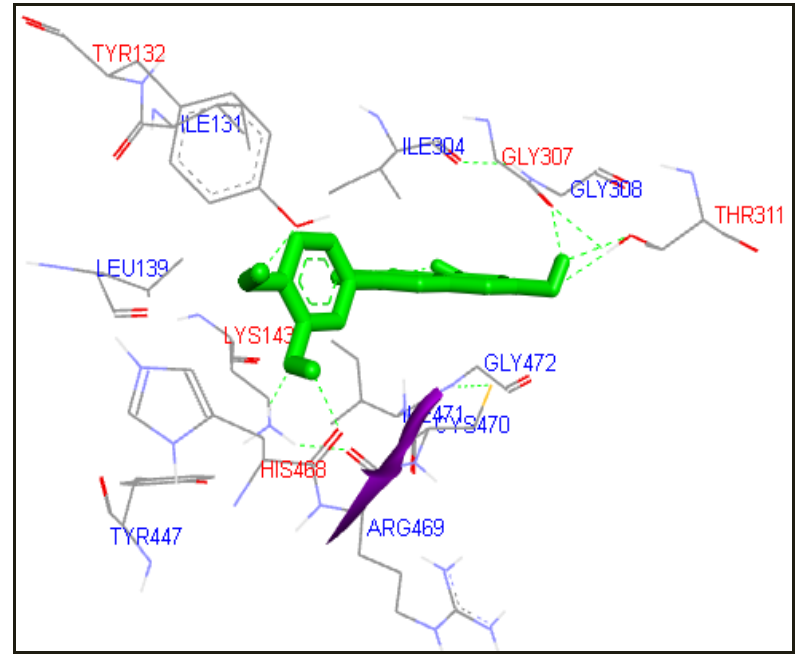

Fig. 7: Docked complex showing Quercetin with lanosterol $14 \alpha$ demethylase

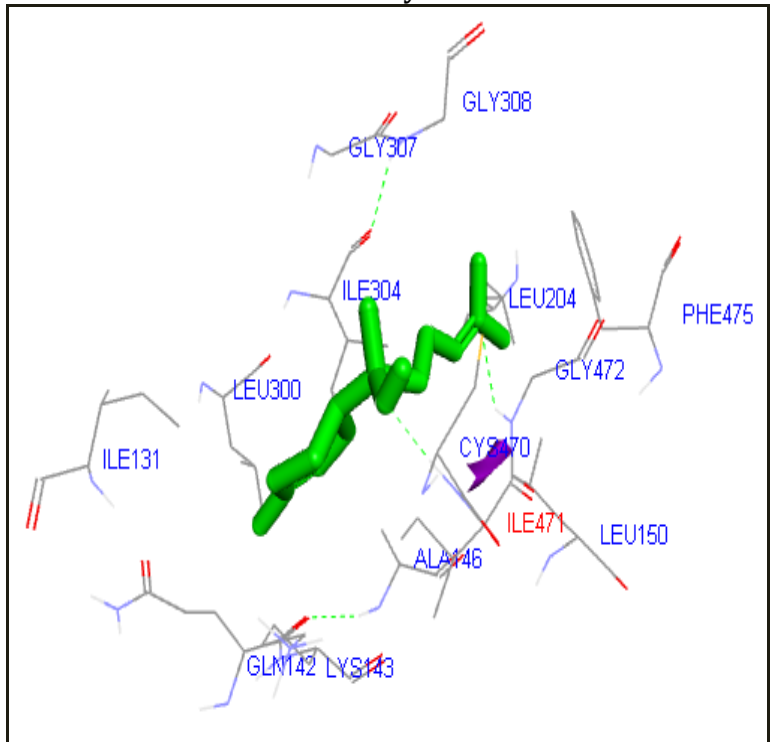

Fig. 9: Docked complex showing alpha-bisabolol with lanosterol $14 \alpha$-demethylase

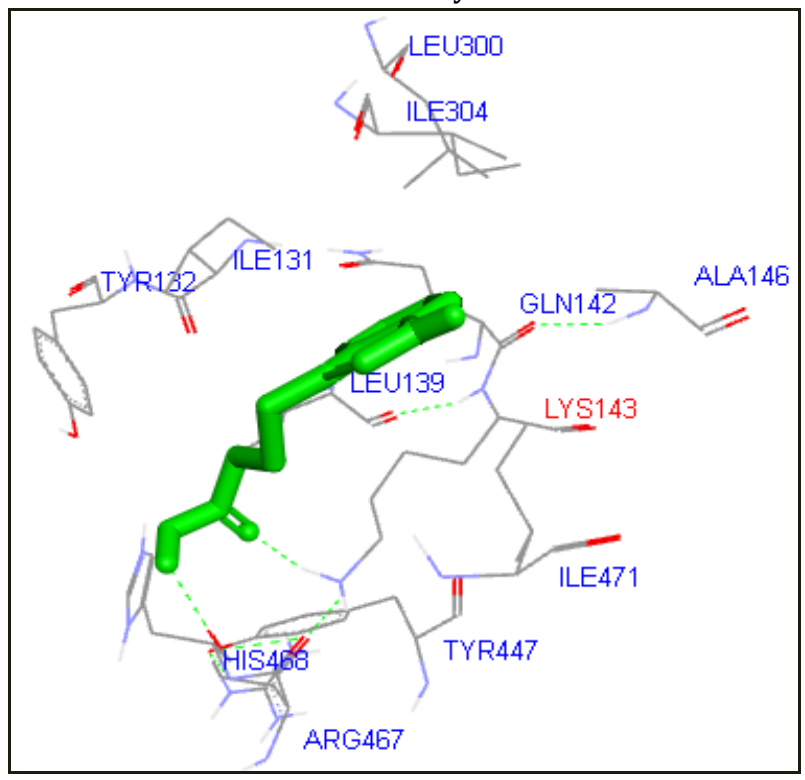

Fig. 11: Docked complex showing Indole-3-butyric acid with lanosterol $14 \alpha$-demethylase

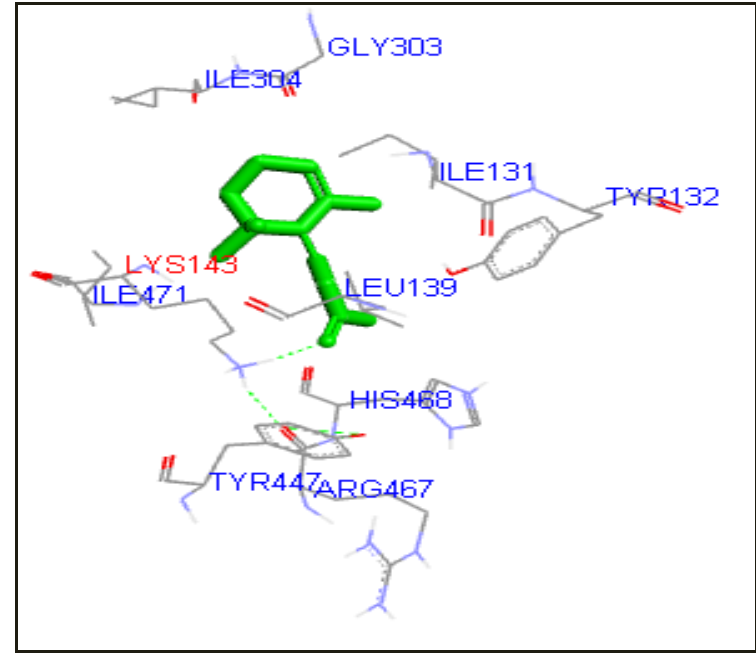

Fig. 8: Docked complex showing Betaionone with lanosterol $14 \alpha$ demethylase

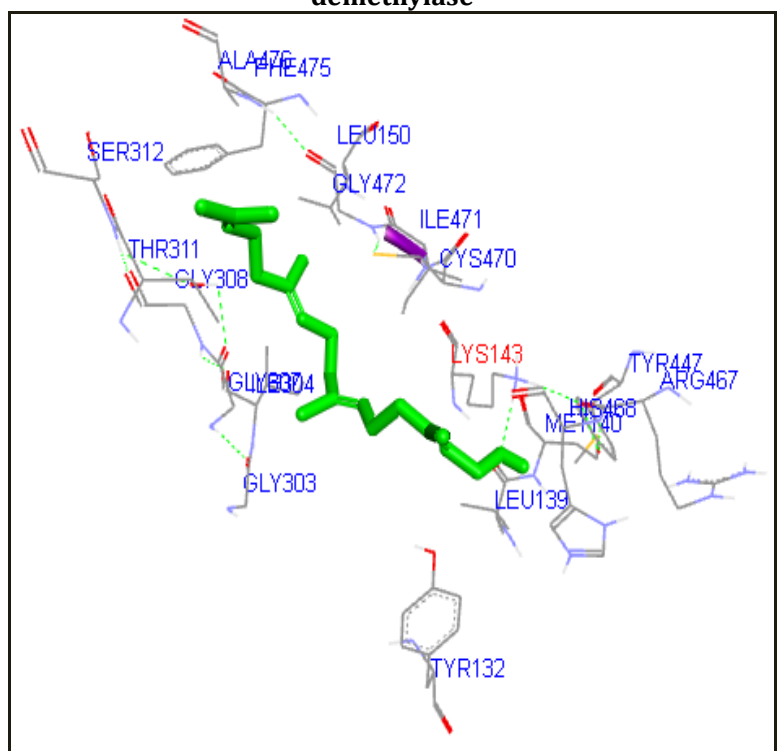

Fig. 10: Docked complex showing geranyl geranoil with lanostero $14 \alpha$-demethylase

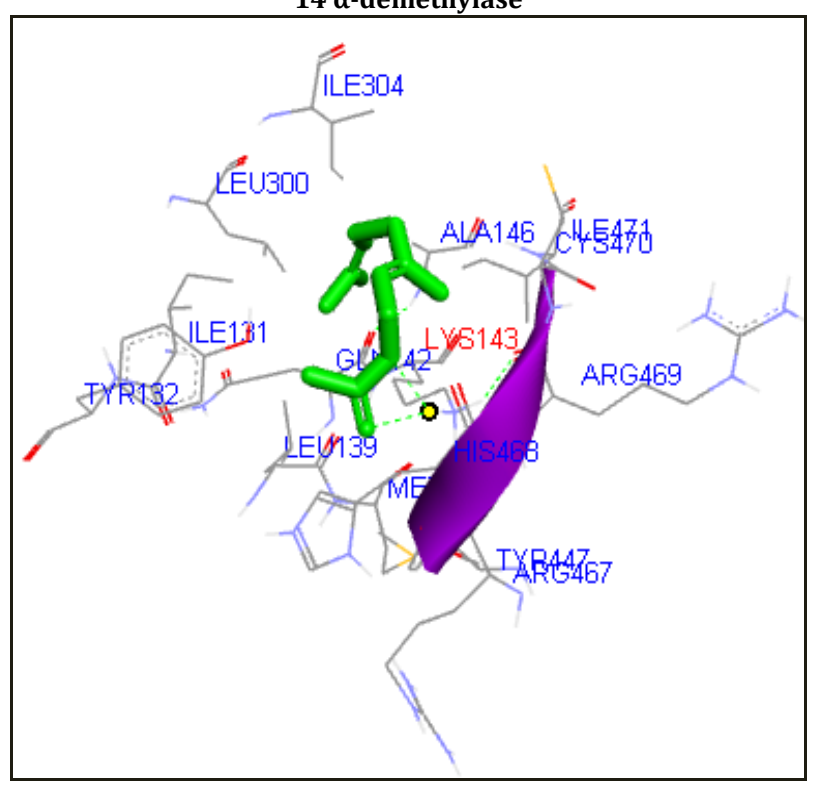

Fig. 12: Docked complex showing Geranylacetate with lanosterol 14 $\alpha$-demethylase 


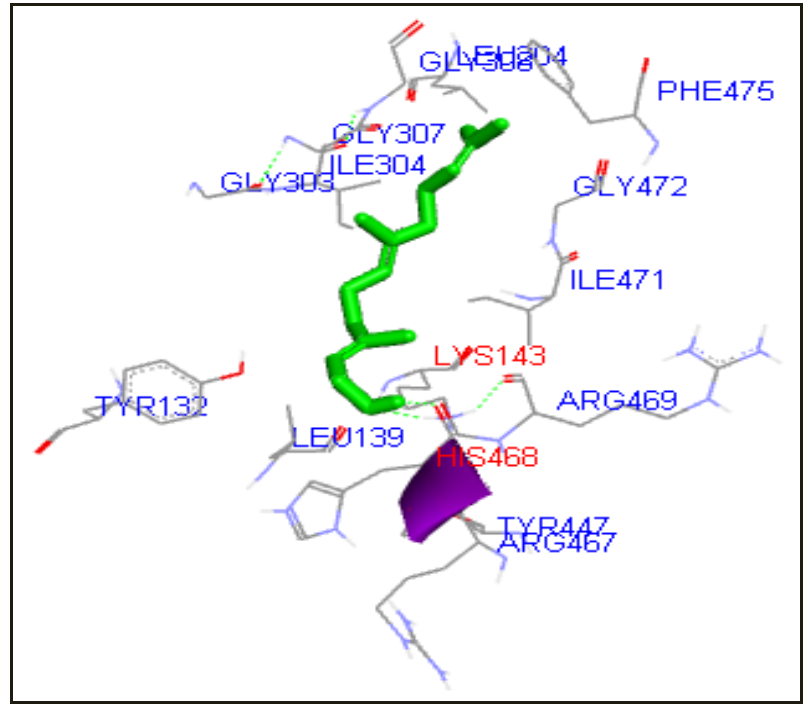

Fig. 13: Docked complex showing Farnesol with lanosterol $14 \alpha-$ demethylase

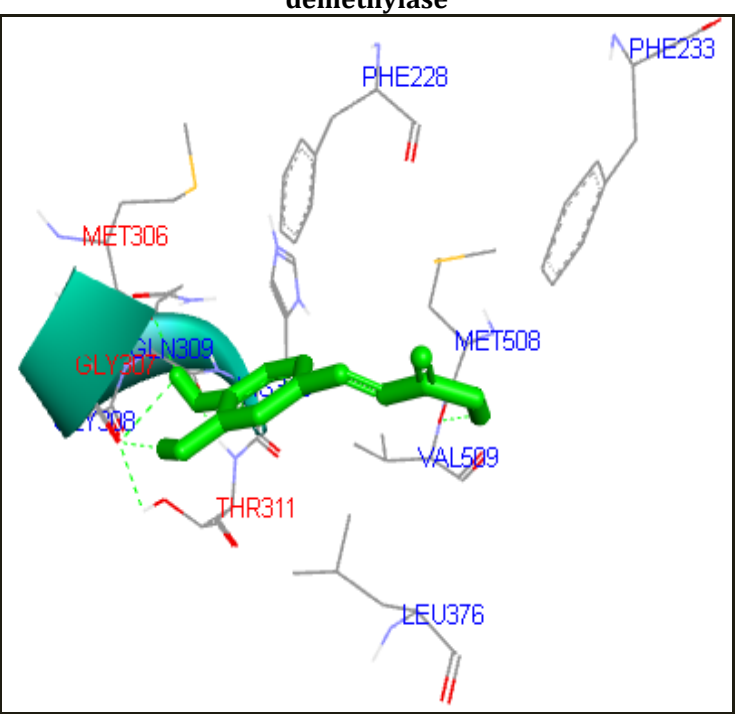

Fig. 15: Docked complex showing caffeic-acid with lanosterol 14 $\alpha$-demethylase

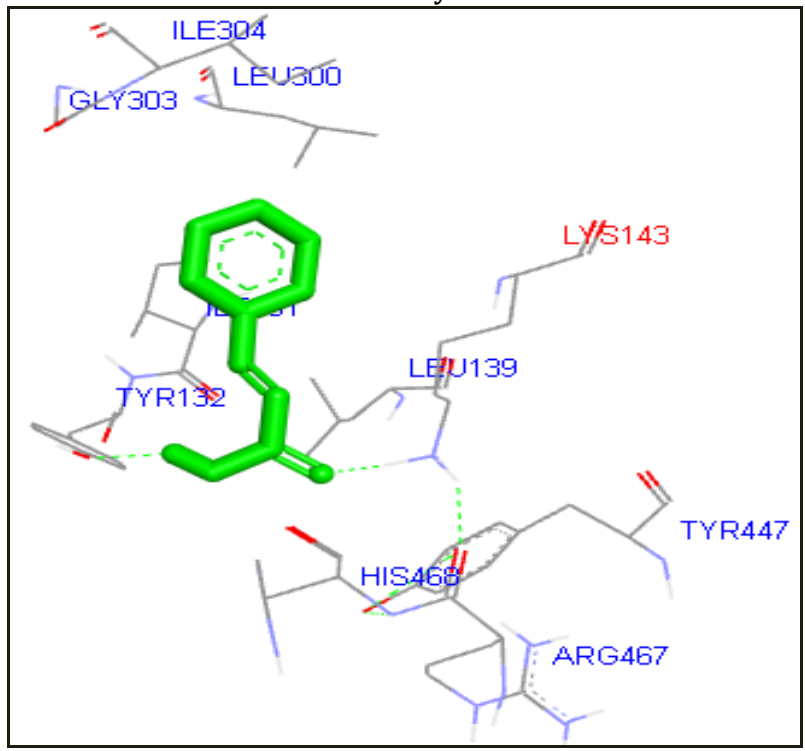

Fig. 17: Docked complex showing cinnamic-acid with lanostero $14 \alpha$-demethylase

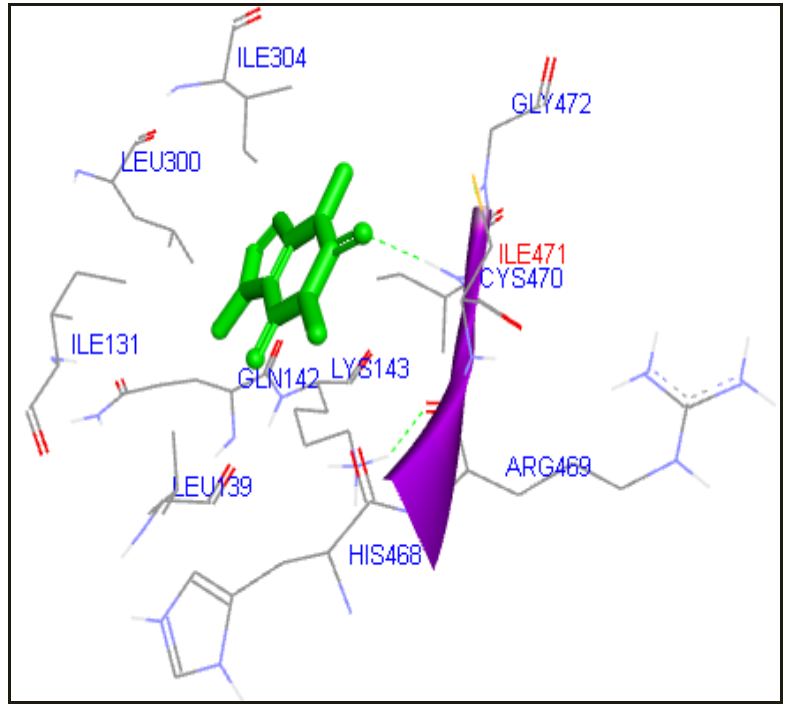

Fig. 14: Docked complex showing caffeine with lanosterol $14 \alpha-$ demethylase

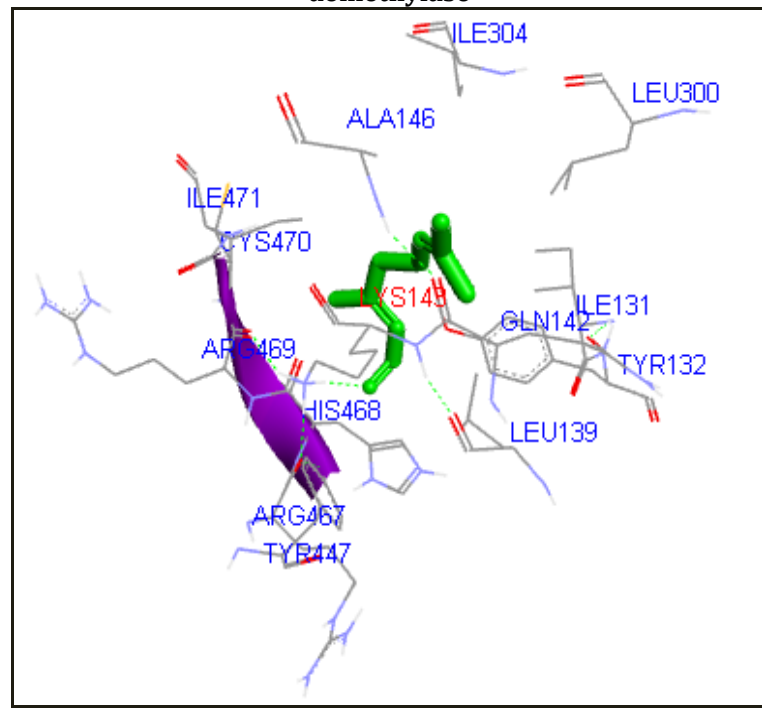

Fig. 16: Docked complex showing citral with lanosterol $14 \alpha$ demethylase

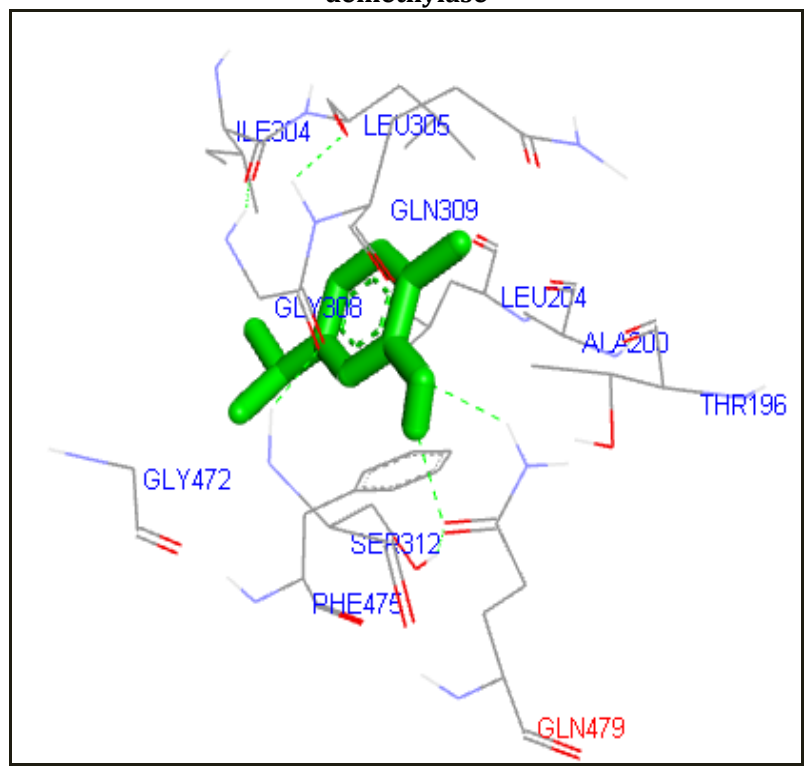

Fig. 18: Docked complex showing carvacrol with lanosterol $14 \alpha$ demethylase 


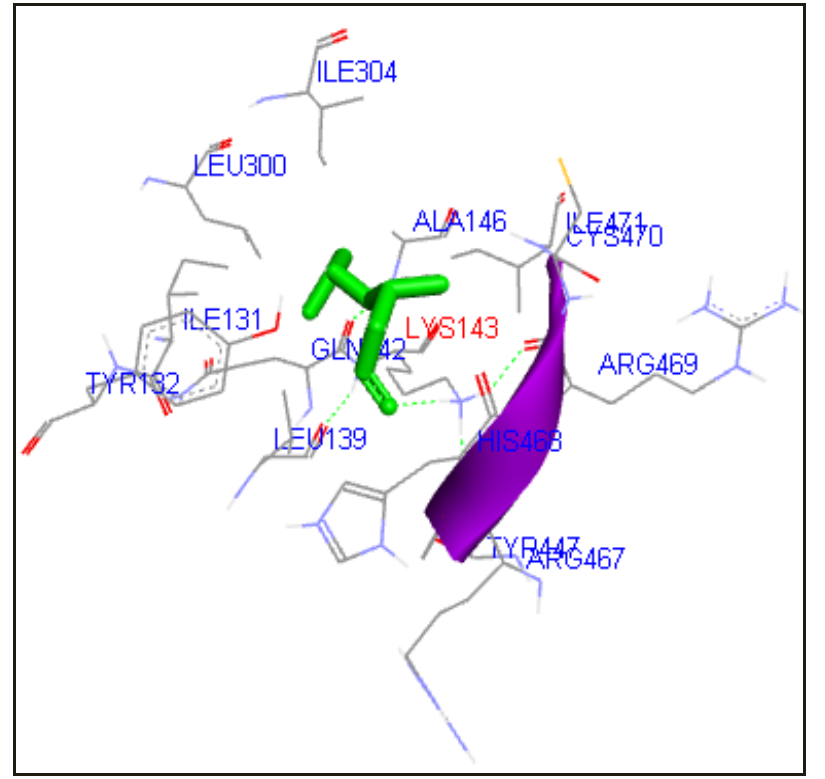

Fig. 19: Docked complex showing citronellol with lanosterol $14 \alpha$ demethylase

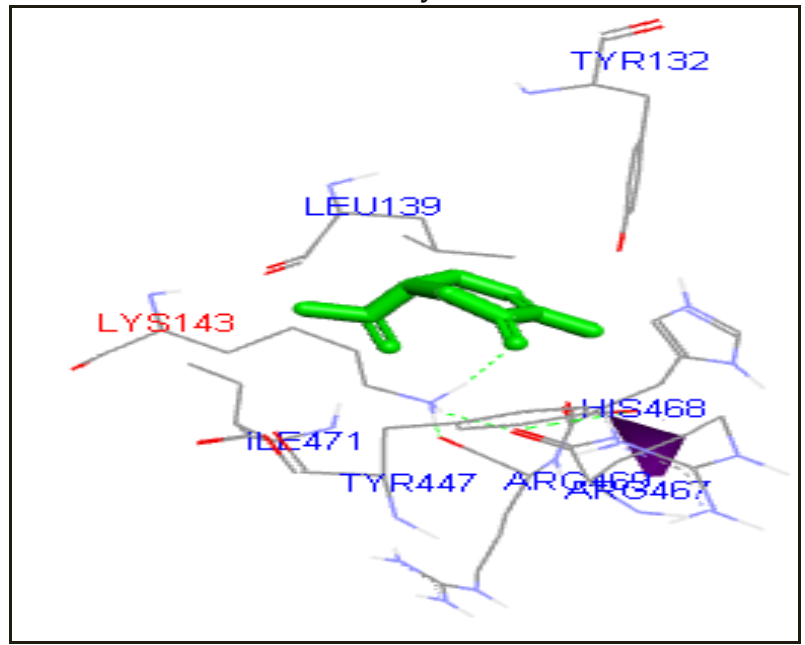

Fig. 21: Docked complex showing carvone with lanosterol $14 \alpha$ demethylase

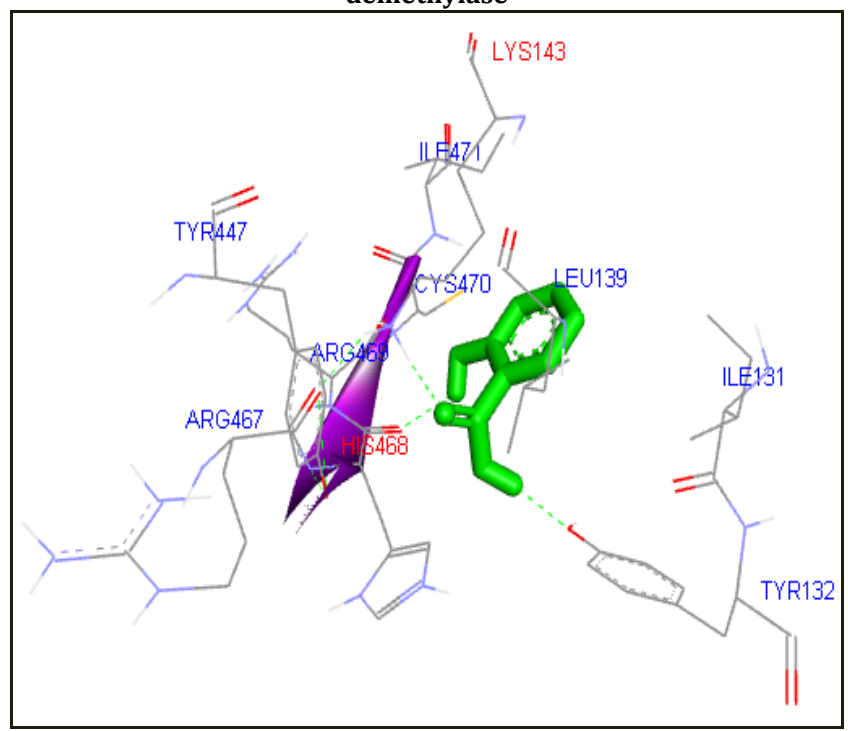

Fig. 23: Docked complex showing Salicylic-acid with lanosterol 14 $\alpha$-demethylase

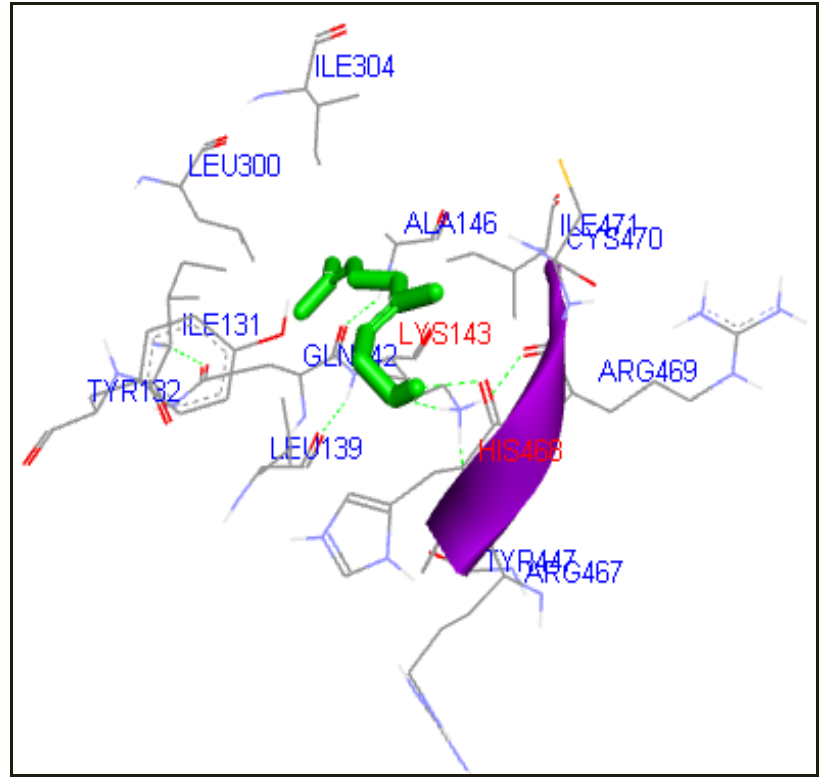

Fig. 20: Docked complex showing geraniol with lanosterol $14 \alpha$ demethylase

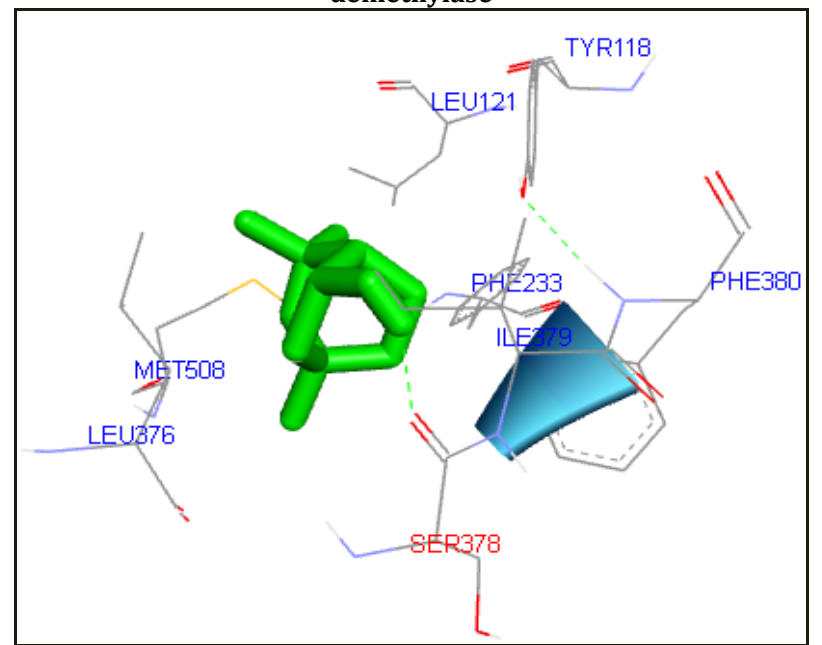

Fig. 22: Docked complex showing 1-8 cineole with lanosterol $14 \alpha$ demethylase

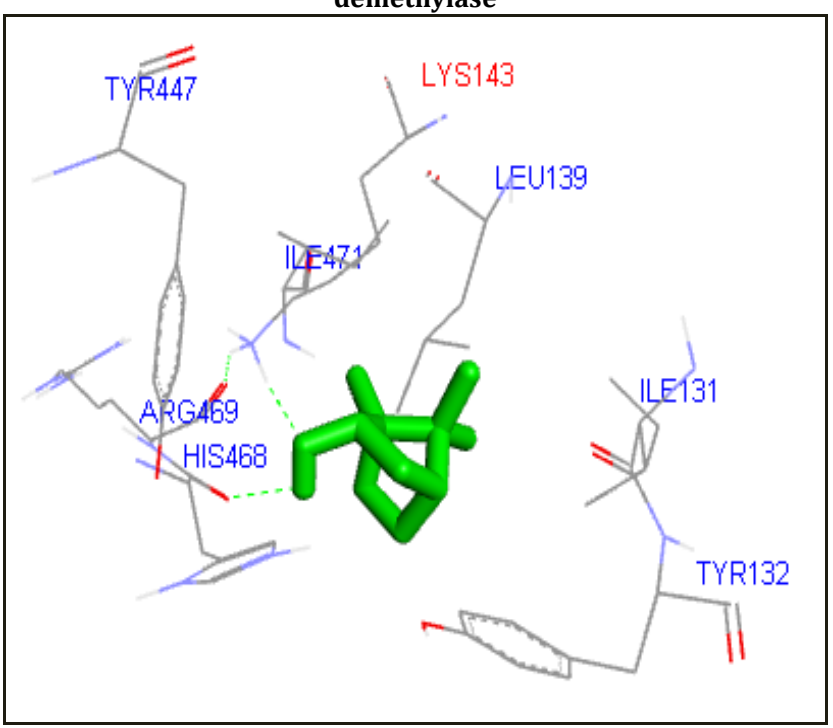

Fig. 24: Docked complex showing Borneol with lanosterol $14 \alpha$ demethylase 


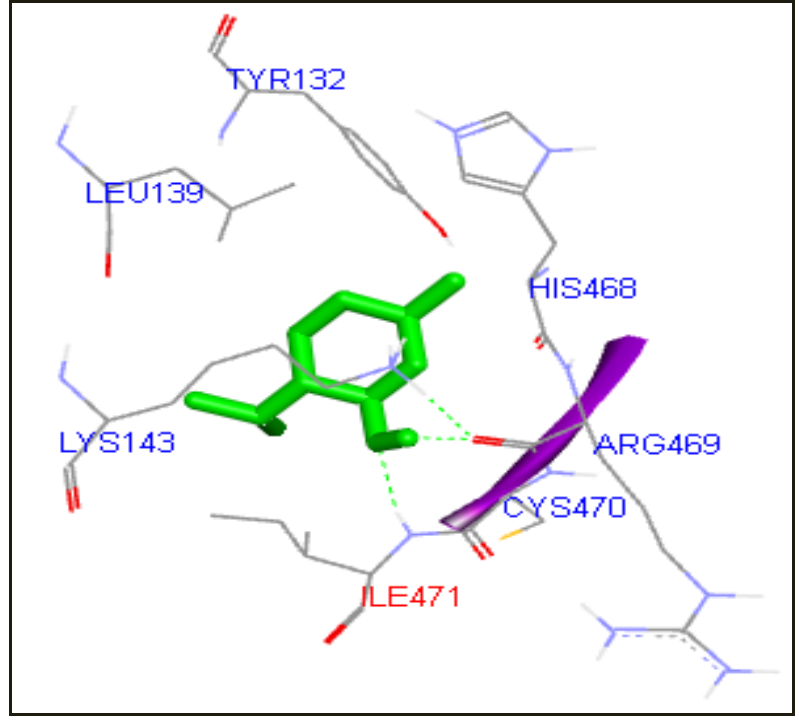

Fig. 25: Docked complex showing Menthol with lanosterol $14 \alpha$ demethylase

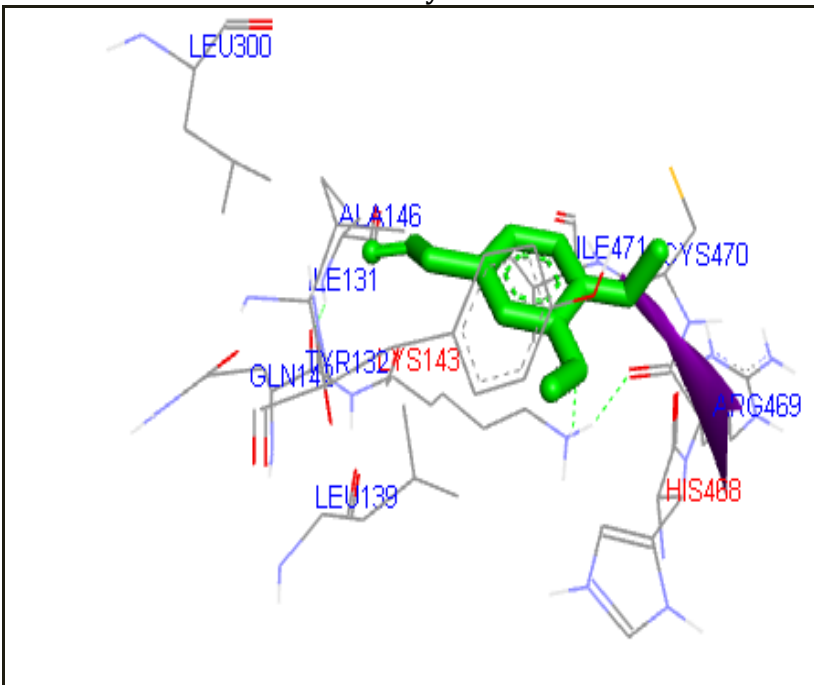

Fig. 27: Docked complex showing Methyleugenol with lanosterol $14 \alpha$-demethylase

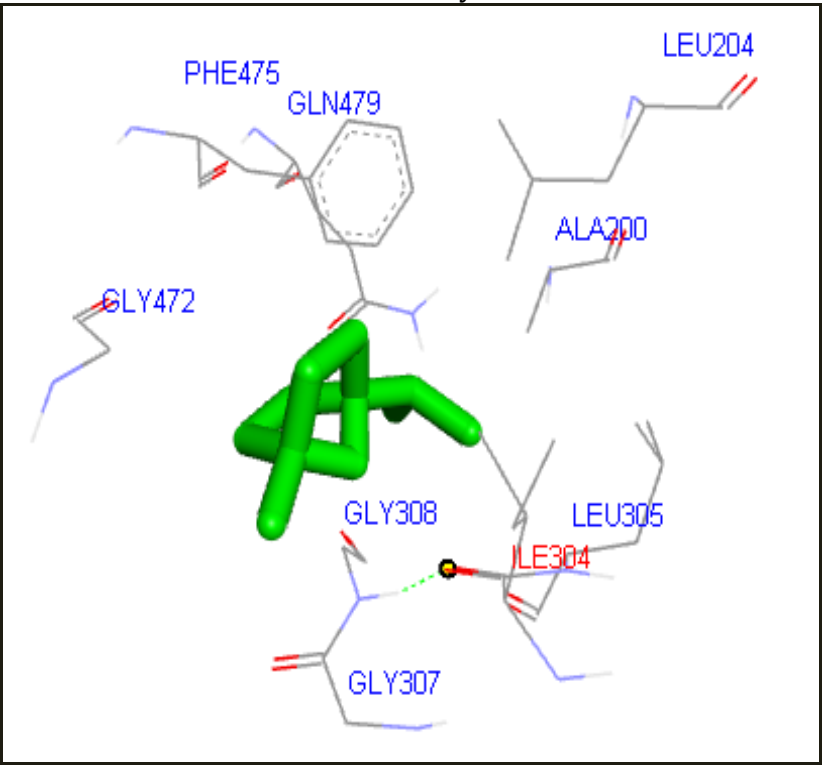

Fig. 29: Docked complex showing 1,4-cineole with lanosterol 14 $\alpha$-demethylase

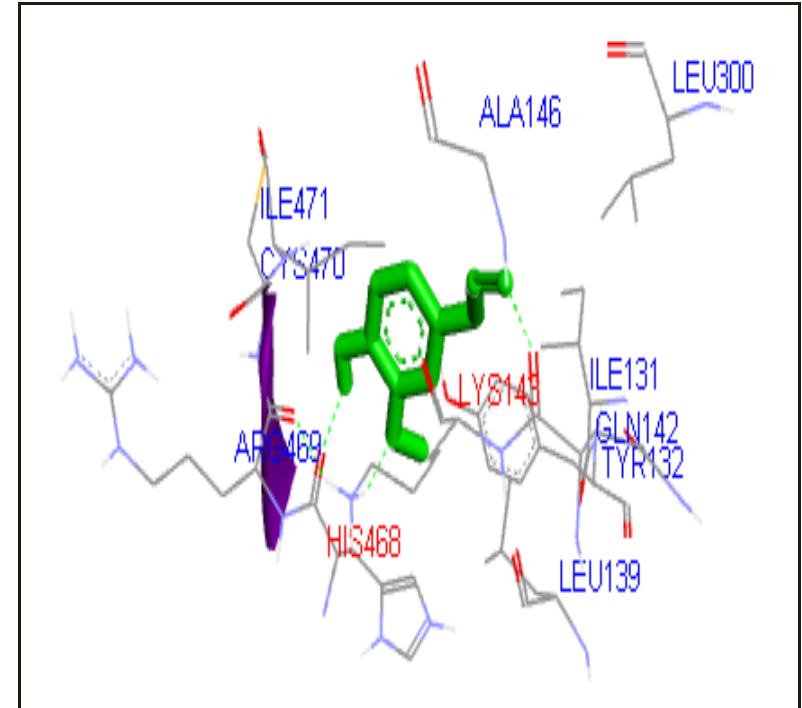

Fig. 26: Docked complex showing eugenol with lanosterol $14 \alpha$ demethylase

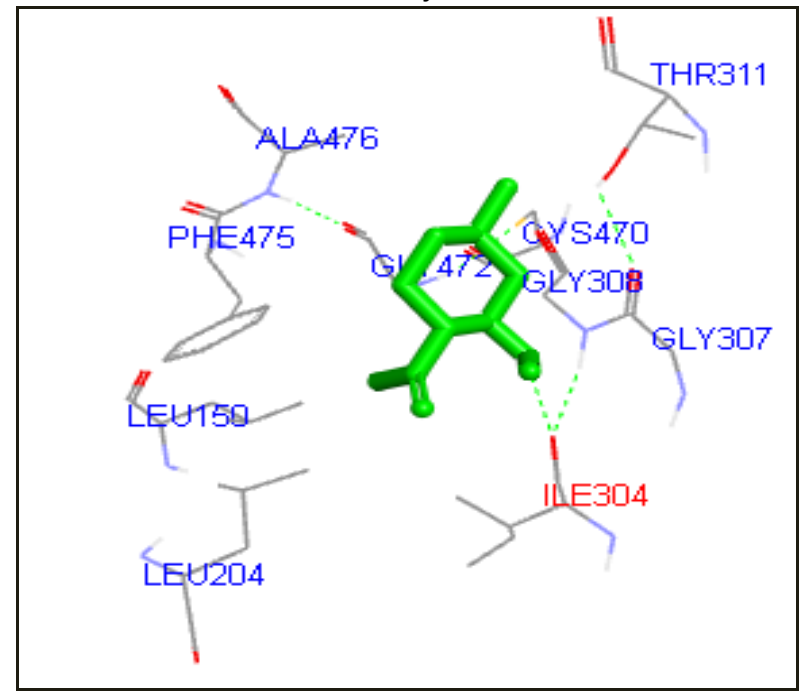

Fig. 28: Docked complex showing Isopulegol with lanosterol $14 \alpha$ demethylase

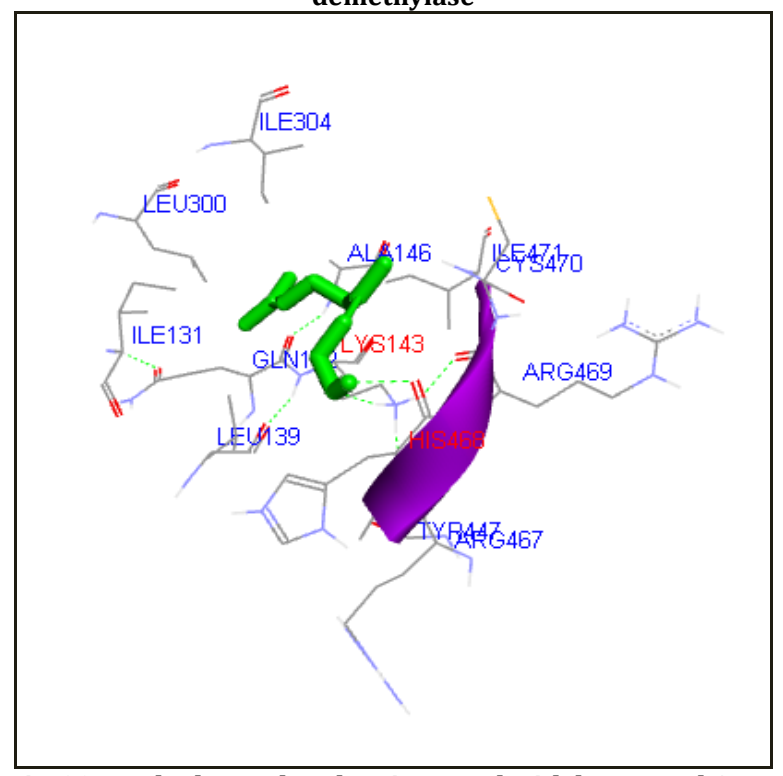

Fig. 30: Docked complex showing nerol with lanosterol $14 \alpha$ demethylase 


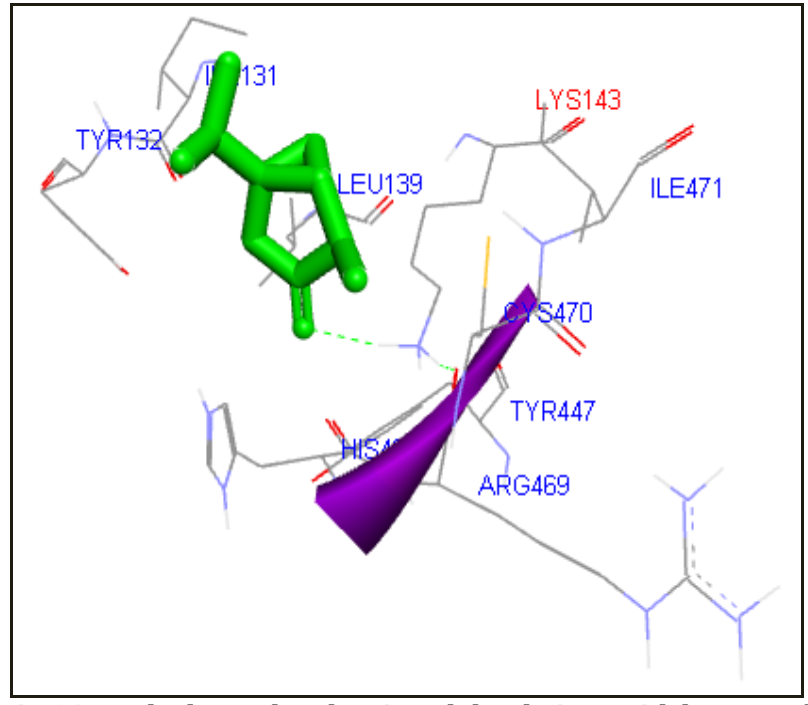

Fig. 31: Docked complex showing Alpha-thujone with lanostero $14 \alpha$-demethylase

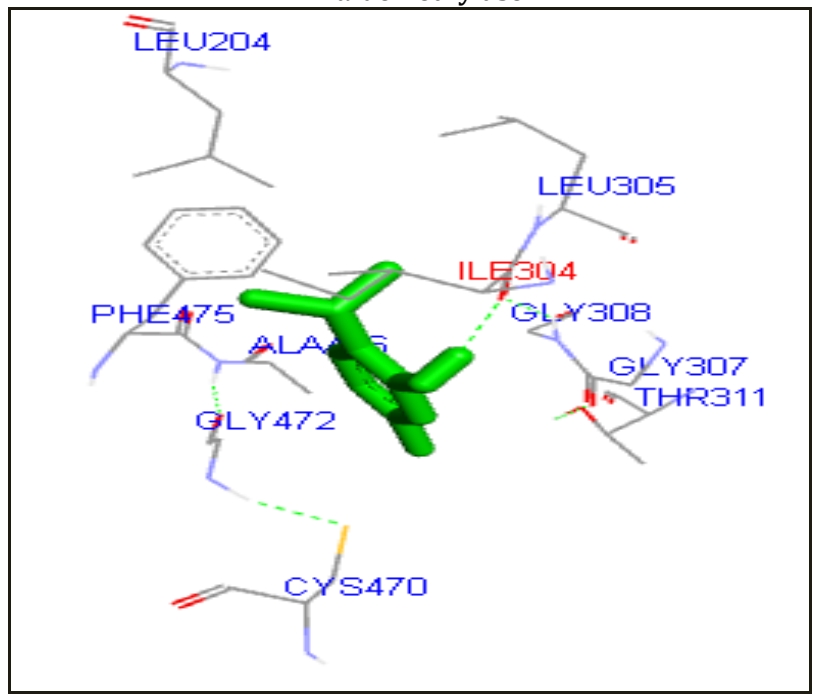

Fig. 33: Docked complex showing thymol with lanosterol $14 \alpha$ demethylase

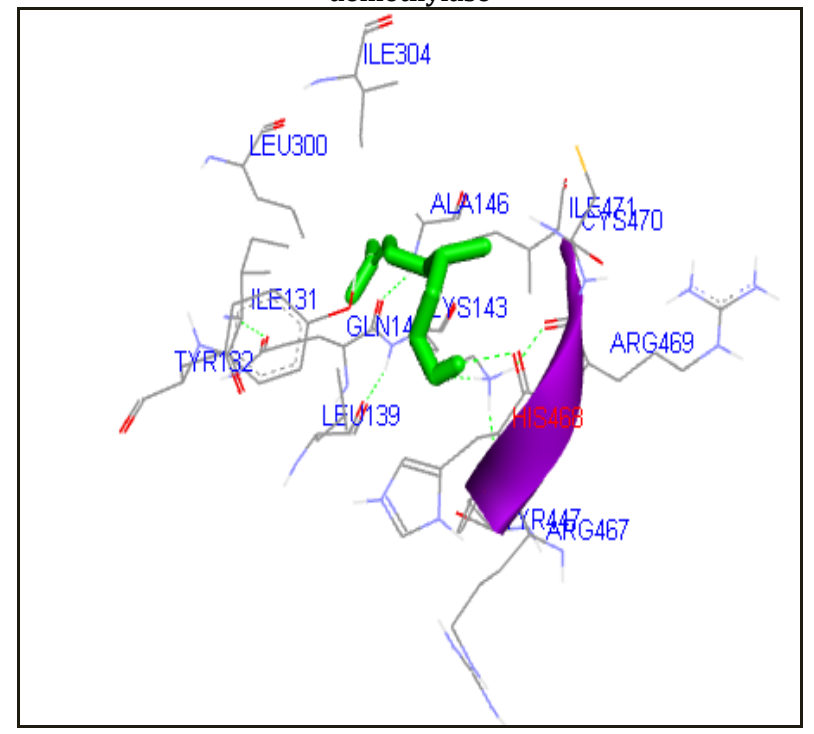

Fig. 35: Docked complex showing betacitronellol with lanosterol $14 \alpha$-demethylase

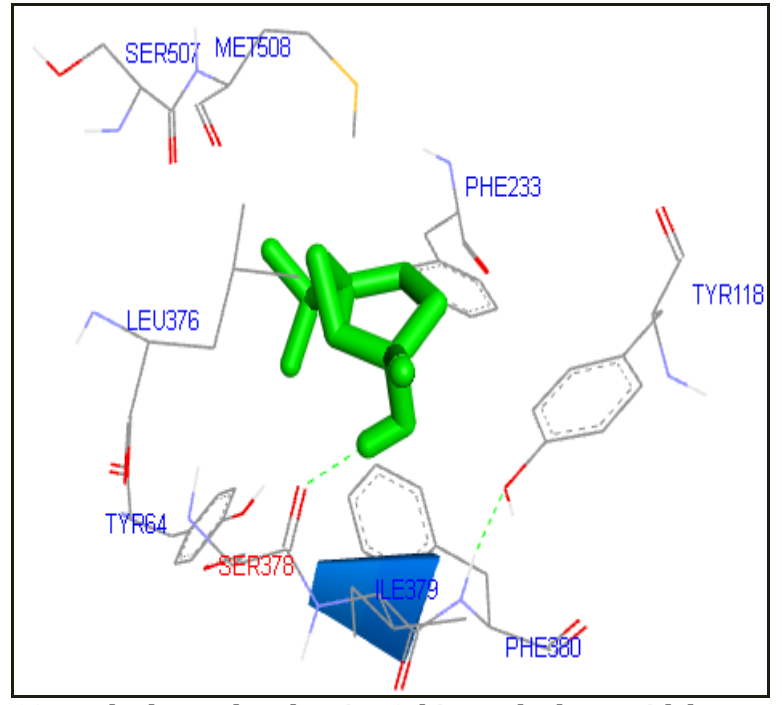

Fig. 32: Docked complex showing Sabinene-hydrate with lanosterol

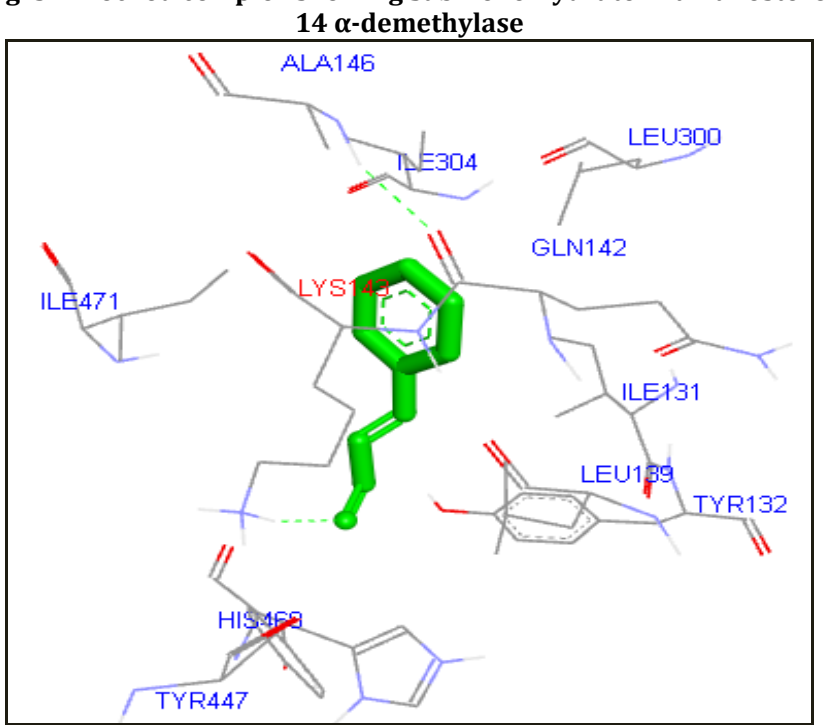

Fig. 34: Docked complex showing cinnamaldehyde with lanosterol $14 \alpha$-demethylase

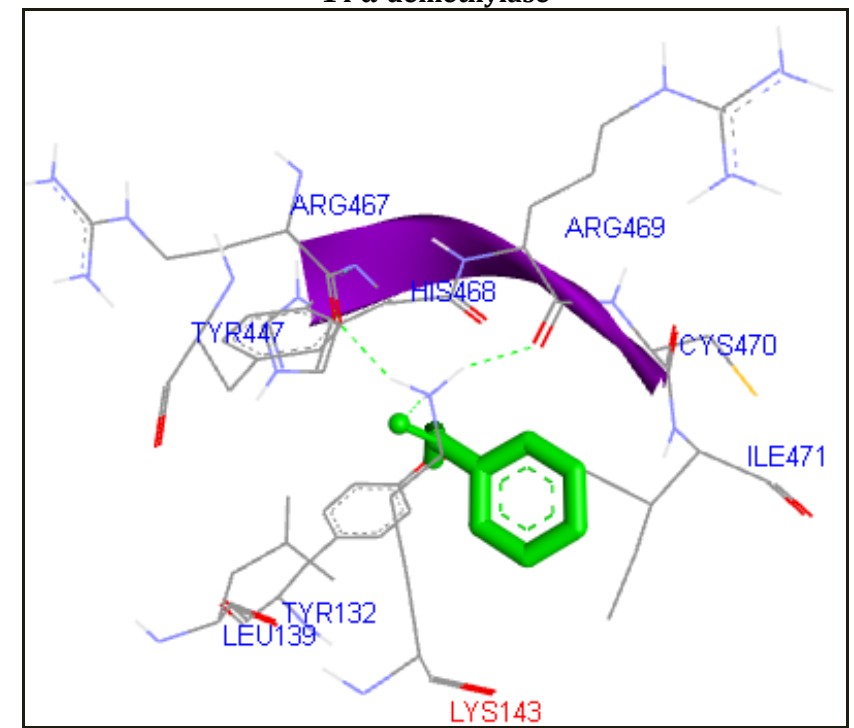

Fig. 36: Docked complex showing nicotinic-acid with lanosterol $14 \alpha$ demethylase 


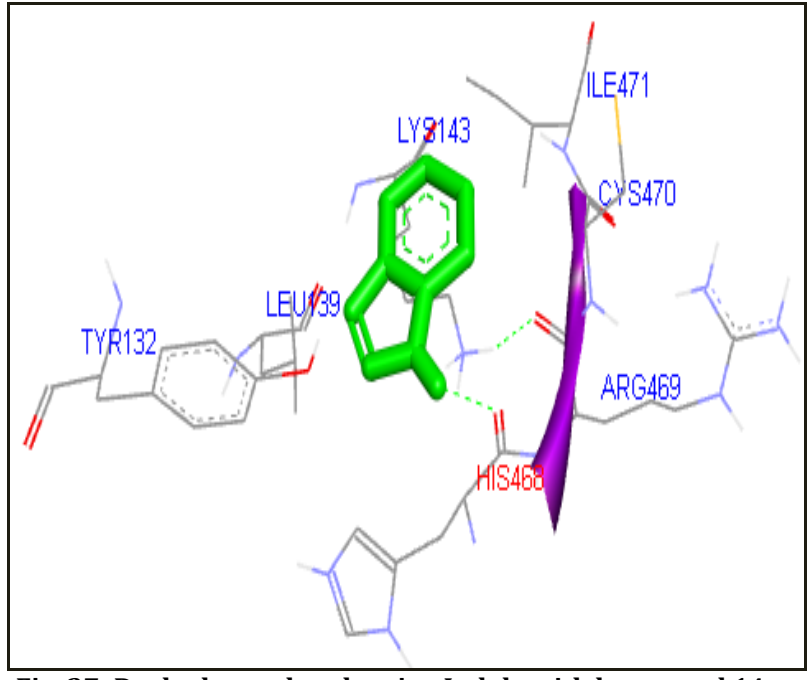

Fig. 37: Docked complex showing Indole with lanosterol $14 \alpha-$ demethylase

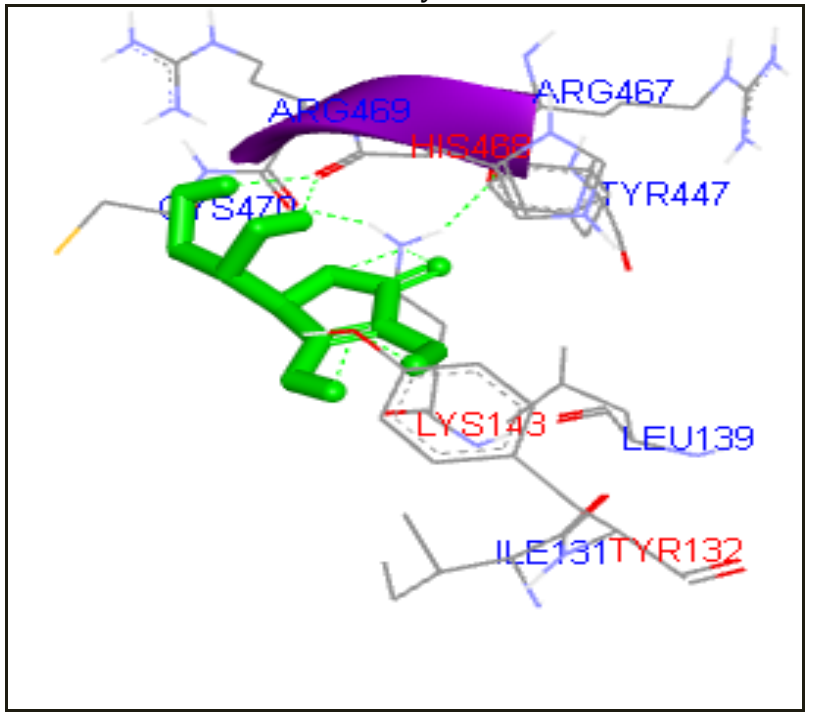

Fig. 39: Docked complex showing ascorbic-acid with lanosterol 14 $\alpha$-demethylase

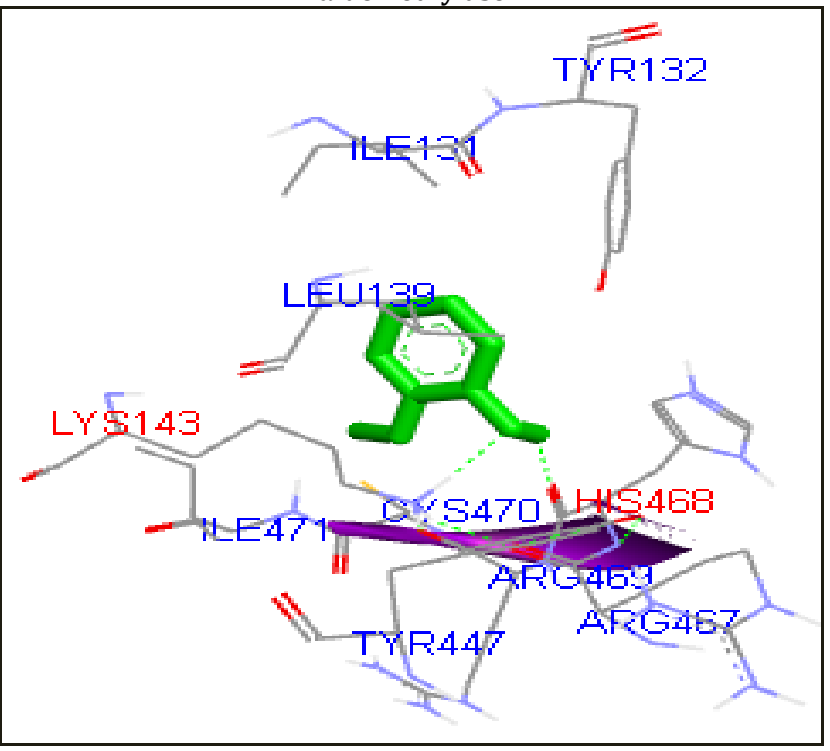

Fig. 41: Docked complex showing guaiacol with lanosterol $14 \alpha-$ demethylase

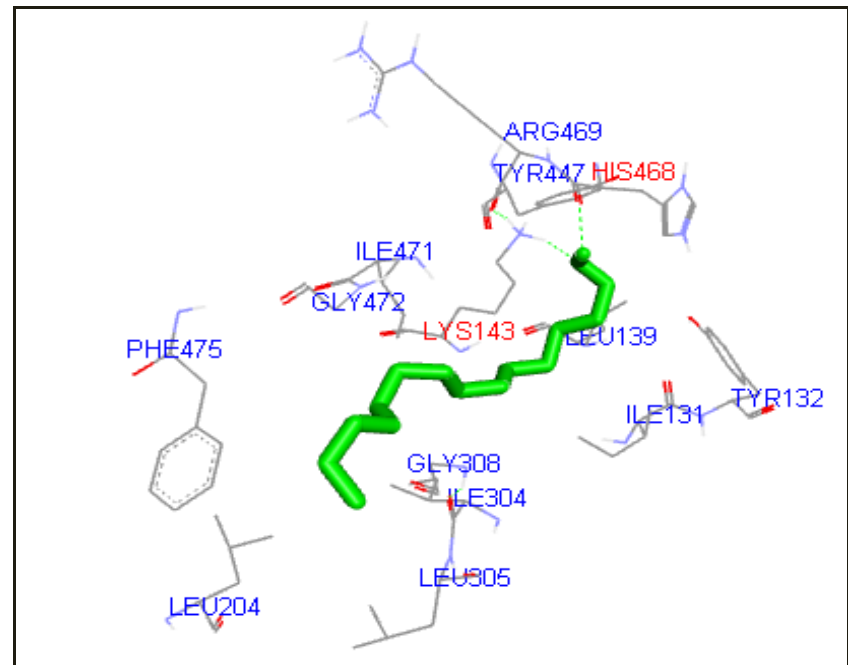

Fig. 38: Docked complex showing 1-tetradecanol with lanosterol 14 $\alpha$-demethylase

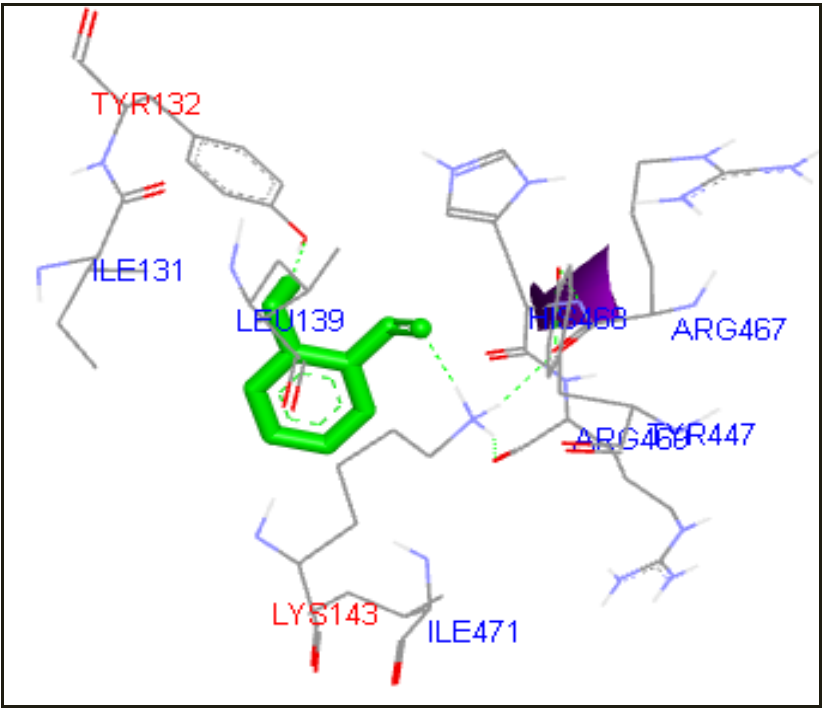

Fig. 40: Docked complex showing Salicylaldehyde with lanosterol 14 $\alpha$-demethylase

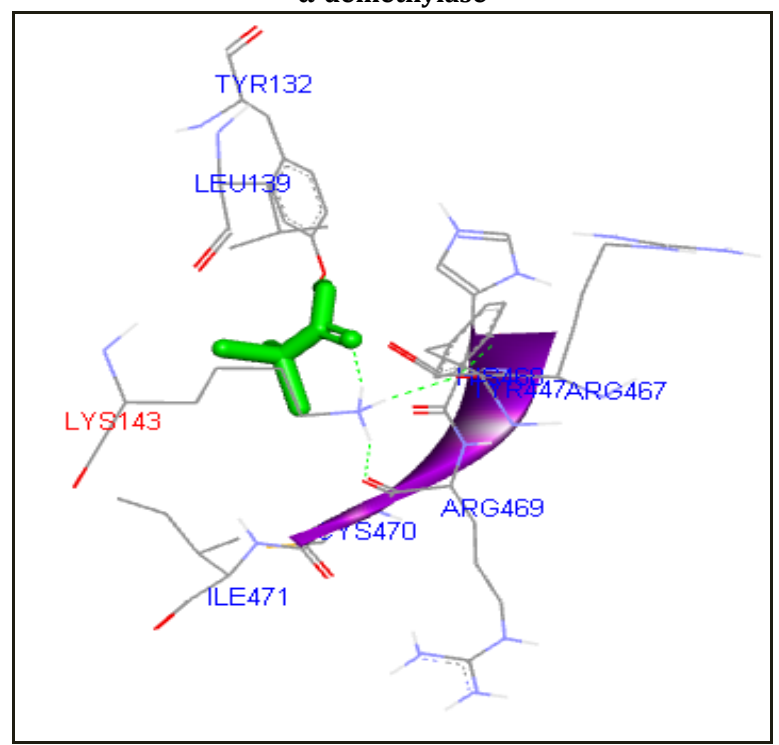

Fig. 42: Docked complex showing trichloroacetic-acid with lanosterol $14 \alpha$-demethylase 


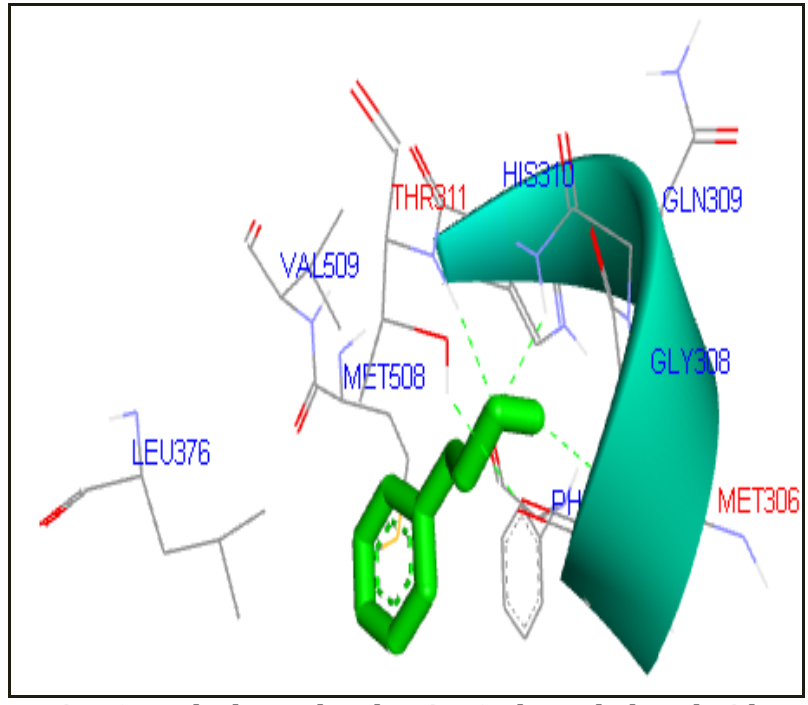

Fig. 43: Docked complex showing 2-phenoylethanol with lanosterol $14 \alpha$-demethylase

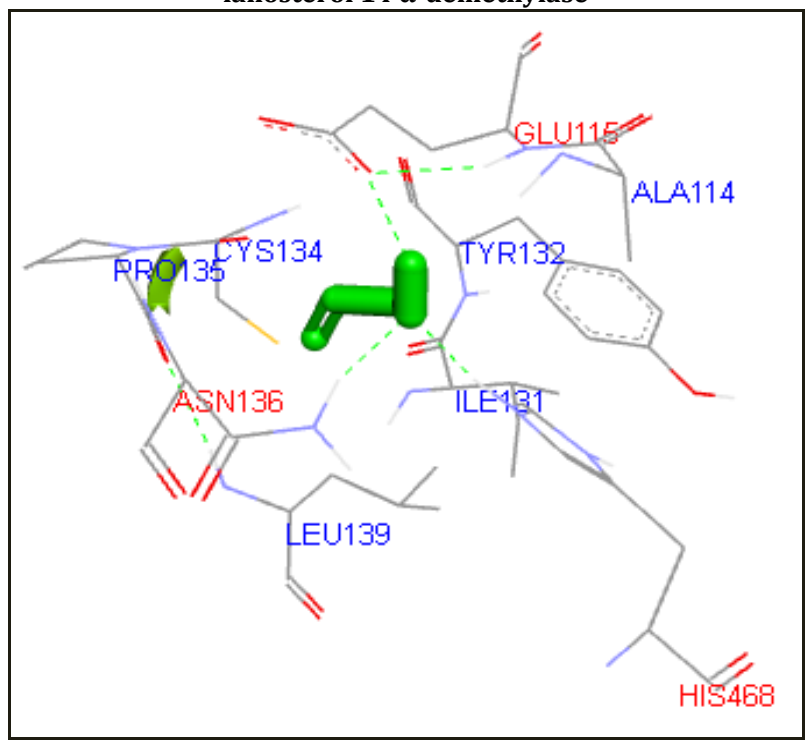

Fig. 45: Docked complex showing allyl-alcohol with lanosterol 14 $\alpha$-demethylase

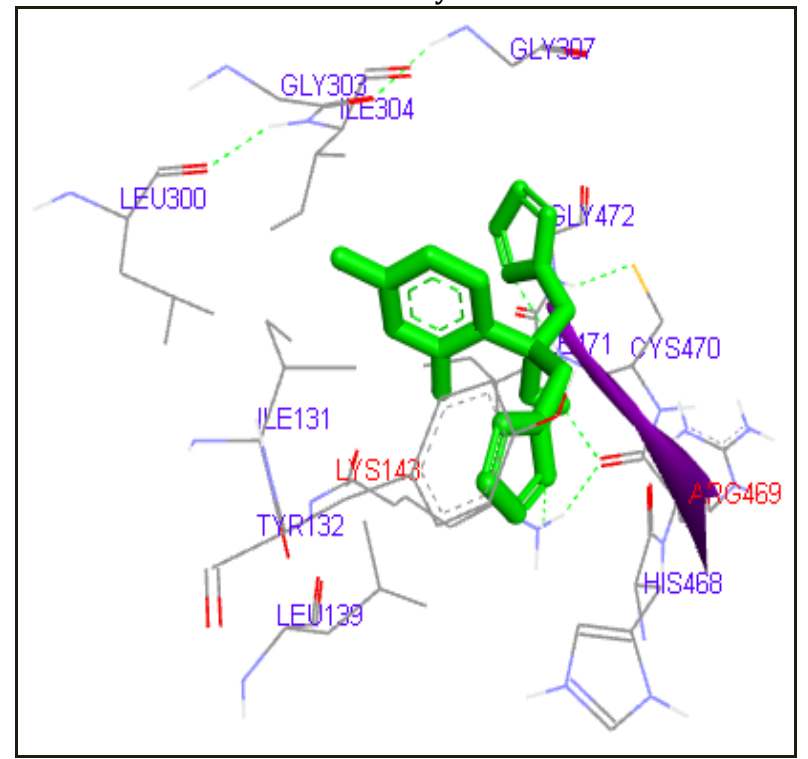

Fig. 47: Docked complex showing fluconazole with lanosterol 14 $\alpha$-demethylase

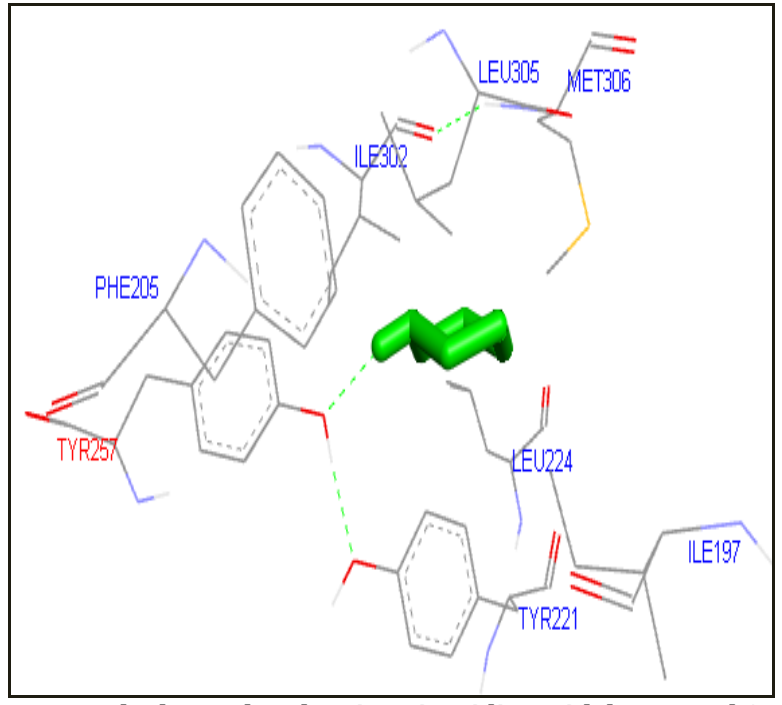

Fig. 44: Docked complex showing piperidine with lanosterol $14 \alpha$ demethylase

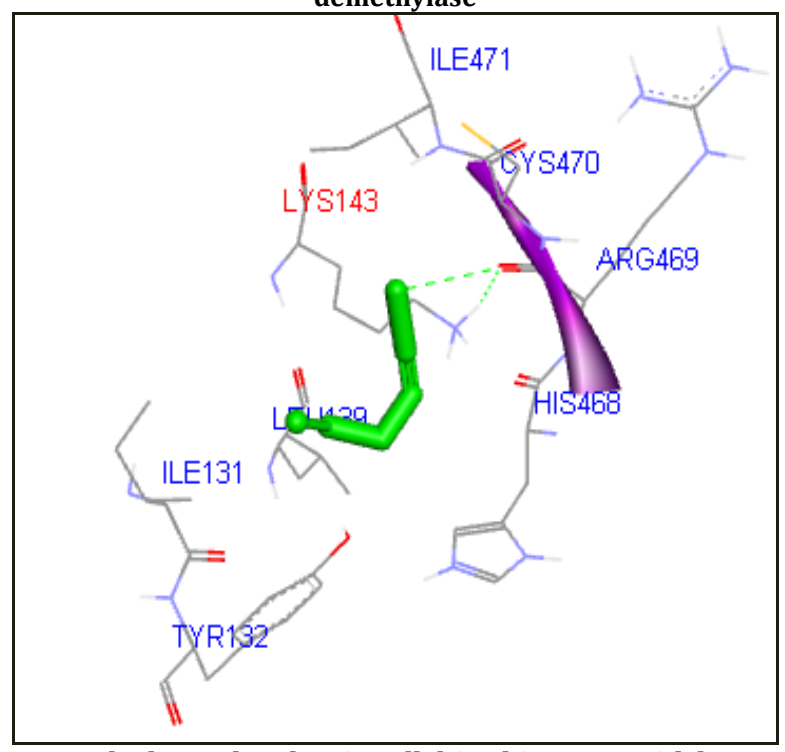

Fig. 46: Docked complex showing allyl-isothiocynate with lanosterol

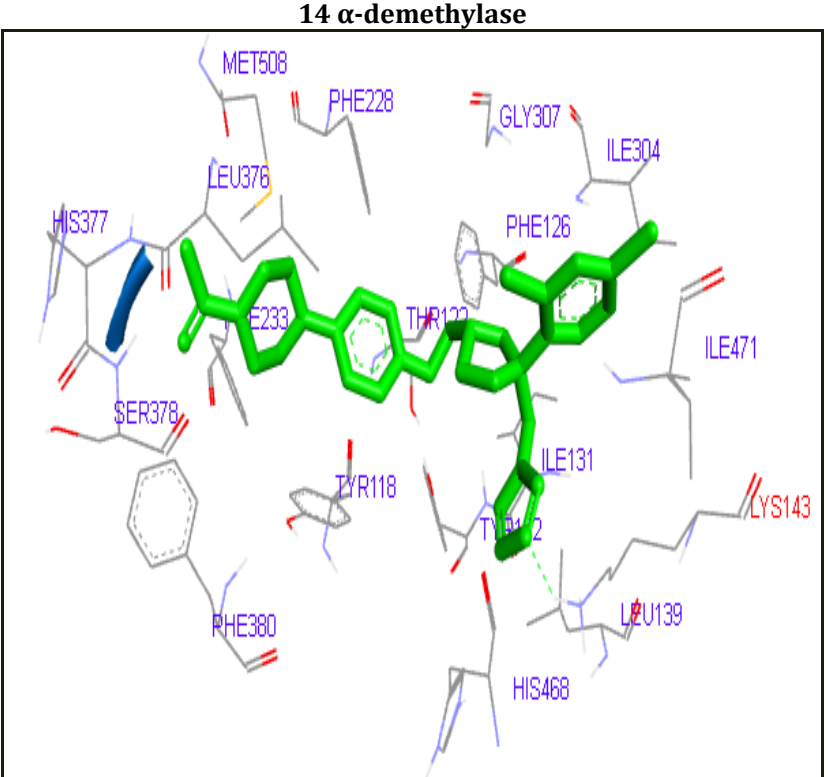

Fig. 48: Docked complex showing ketoconazole with lanosterol $14 \alpha$ demethylase 


\section{DISCUSSION}

Ergosterol biosynthesis is considered as an antifungal target because ergosterol is vital for the survival of fungal cell [16-18]. Lanosterol $14 \alpha$-demethylase catalyses the conversion of lanosterol to ergosterol [18]. For example, Fluconazole can inhibit ergosterol biosynthesis by inhibiting the activity of lanosterol $14 \alpha-$ demethylase enzyme. Amphoterin B can inhibit ergosterol polymerization and creates hole in the ergosterol membrane and leads to the leak of ions from the cell [18]. These two drugs are very important, however, these drugs has various side effects. For example, Amphoterin B has a serious nephrotoxicity effect, while Fluconazole is fungi static and can affect the estrogen synthesis [19]. Drug resistance and mutation in ergosterol biosynthetic gene ERG11 is also reported [20]. There are few other drugs which are known to be ergosterol inhibitors like Ketoconazole. Considering the side effect of these molecules, there some studies where people have explored the potential of plant molecules as ergosterol inhibitors. Rajput and Karuppayil (2013) has studied the efficacy of twenty-five plant molecules, Cinnamaldehyde, Piperidine, Indole, Furfuraldehyde, Citral, Beta-Pinene, Salicylic Acid, Guaiacol, Cymene, Caffeine, Camphene, Citronellol, Geraniol, Geranylacetate, AlphaPinene, Carvone, Linalool, Thujone, Bisabolol, Jasmonate, Isopulegol, Limonene, 1,4-Cineole, 1,8-Cineole, and Menthol, on the synthesis of ergosterol in the human pathogen and Candida albicans [21]. Out of 25 molecules studied six molecules, they have identified as inhibitors of ergosterol. But their study has not defined what these molecules targeted in ergosterol biosynthetic pathway. Ahmad et al., (2011) studied the Fungicidal activity of Thymol and Carvacrol; they found that both these molecules showed fungicidal activity by inhibiting ergosterol biosynthesis [22]. Prasanna et al., (2014) have studied 25 molecules which are found in plants used in Siddha medicine, Aurantiamide Acetate, B-Sitosterol, Kaempferol, Clitorin, Mauritianin, Nicotiflorin, Vitexdoin A, Vitedoamine B, Vitexin, Betulinic Acid, Oleanolic Acid, Caryophyllene Oxide, Daturamalakin B, Hyoscyamine, Phenowithanolide, Withametelin, Scopolamine, 1, 6-Heptadiene-3, 5-Dione, 5-Hydroxyl-1,7-Bis 4, 6-Heptadiene-3-One, Tetrahydroxycurcumin, Curcumin, Demethoxycurcumin, Bisdemethoxy-curcumin including Fluconazole and Ketoconazole and they have given the binding energy of these molecules. Their study revealed that clitorin, mauritianin, and kaempferol bound to lanosterol 14 a-demethylase by hydrogen bonding and hydrophobic interaction [23].

Considering the efficacy of plant molecules as inhibitors of ergosterol biosynthesis, we wanted to know whether these plant molecules may interact with lanosterol $14 \alpha$-demethylase enzyme or not. Our study suggests that out of sixty plant molecules, forty-eight molecules are showing binding with lanosterol $14 \alpha$-demethylase (table 1). Even though molecules like Alpha-pinene, Beta-pinene, Camphene, and Limonene are showing ergosterol inhibition in the study of Rajput and Karuppayil (2013), in our study they were not found to be interacting with lanosterol $14 \alpha$-demethylase. So it means that they may have different targets other than lanosterol $14 \alpha$-demethylase (table 1).

We compared our docking results with Ketoconazole to find the similarity among our molecules and standard drug. Interestingly, we found that out of 48 docked molecules, 14 molecules Caryophylleneoxide, Betaionone, Indole-3-butyric-acid, Geranylgeranoil, Geranylacetate, Citral, Cinnamic-acid, Citronellol, Carvone, Borneol, Alpha-thujone, Cinnamaldehyde, Nicotinic-acid, and Allyl-alcohol showed similar interacting residue LYS143 as that of Ketoconazole (table 2). Out of 14 molecules, Caryophyllene-oxide, Betaionone, Indole-3-butyric-acid, Geranylgeranoil, Geranylacetate, Citral, Cinnamic-acid, Citronellol, Carvone, Borneol, Alpha-thujone, Cinnamaldehyde, Nicotinic-acid, and Allyl-alcohol, six molecules are already known to inhibit ergosterol biosynthesis. Caryophylleneoxide is known to inhibit the ergosterol biosynthesis [21, 23]. Geranylacetate, Citral, Citronellol, Carvone, and Cinnamaldehyde are known to have inhibitory affect against lanosterol $14 \alpha$-demethylase [14]. The above data supports our hypothesis that as Ketoconazole interacting to lanosterol $14 \alpha$-demethylase by LYS143 amino acid and 14 molecules have same binding residue may have similar mode of action. Rest the of eight molecules interacting via the similar
LYS143 residue as that of Ketoconazole may also have same ergosterol inhibiting effect as that of Ketoconazole and may be used as potent antifungal drugs.

More than 300 million people are reported to suffer from a serious fungal infections resulting in over $1,350,000$ deaths [24]. Importance of fungal infections has led to a remarkable rise in the application of antifungal agents for the treatment and prevention of infection. Regrettably, the treatment options are highly limited, as there are few chemical classes represented by existing antifungal drugs [25]. On the contrary, azole groups of drugs are most commonly prescribed drugs for the treatment of Candida albicans infections for more than $30 \mathrm{y}$ although prolonged use of any drugs, causes drug resistance [26-30].

In this study out of sixty plant molecules 12 plant molecules (Alphapinene, Beta-pinene, Camphene, Beta_elemene, Alpha_phellandrene, Eucalyptol, Myrcene, P-cymene, Sabinene, Terpinolene, Gammacadinene, and Limonene) has shown no hydrogen bond formation with the active site of lanosterol $14 \alpha$-demethylase (table 1).

Previous studies state that to interact with ligand, there must have some means of interaction between molecule and protein like hydrogen bond formation [31]. Those 48 plant molecules showing excellent and good binding affinities may inhibit the activity of lanosterol $14 \alpha$-demethylase by binding at its active site. These results may need to be confirmed ergosterol assay.

\section{CONCLUSION}

Molecular Docking studies revealed that out of 60 molecules 48 molecules has shown good binding affinity with lanosterol 1, $4 \alpha-$ demethylase. These molecules may inhibit the activity of lanosterol $14 \alpha$-demethylase and thereby inhibit the ergosterol synthesis in Candida albicans. 14 molecules have shown similar interactions as that of Ketoconazole since they are binding at LYS143. On the other hand, our study also revealed that 12 molecules did not form any hydrogen bond with the predicted model of lanosterol $14 \alpha$ demethylase; however, it doesn't mean that these 12 molecules may have no activity, as previous study shows these molecules also have antifungal activity their target may be different from that of lanosterol $14 \alpha$-demethylase enzyme. From the present study, we conclude that lanosterol $14 \alpha$-demethylase is a good target for antifungal drug,s. Using our 48 docked molecules one can develop new antifungal/potential drugs which are the inhibitors of lanosterol $14 \alpha$-demethylase of Candida albicans. There is a need for in vitro and in vivo studies to cofirm the efficacy of these molecules against Candida albicans pathogenesis.

\section{ACKNOWLEDGMENT}

AKJ is thankful to DY Patil Education Society (Deemed to Be University) Kolhapur for funding support.

\section{AUTHORS CONTRIBUTIONS}

All the authors have contributed equally.

\section{CONFLICT OF INTERESTS}

Authors declare that there is no Conflict of Interest.

\section{REFERENCES}

1. Jacob KS, Ganguly S, Kumar P. Homology model, molecular dynamics simulation and novel pyrazole analogs design of Candida albicans CYP450 lanosterol $14 \alpha$-demethylase, a target enzyme for antifungal therapy. J Biomol Struct Dyn 2016;35:1-18.

2. Denning DW, Bromley MJ. How to bolster the antifungal pipeline. Science 2015;347:1414-6

3. Bard M, Lees ND, Turi T. Sterol synthesis and viability of erg 11 (cytochrome P450 lanosterol demethylase) mutations in Saccharomyces cerevisiae and Candida albicans. Lipids 1993;28:963-7.

4. Ji H, Zhang W, Zhou Y. A three-dimensional model of lanosterol $14 \alpha$-demethylase of Candida albicans and its interaction with azole antifungals. J Med Chem 2000;43:2493-12.

5. Lamb DC, Kelly DE, Venkateswarlu K. Generation of a complete, soluble, and catalytically active sterol $14 \alpha$-demethylase-reductase complex. Biochemistry 1999;38:8733-8. 
6. Trzaskos JM, Fischer RT, Favata MF. Mechanistic studies of lanosterol C-32 demethylation. Conditions which promote oxysterol intermediate accumulation during the demethylation process. J Bio Chem 1986;261:16937-42.

7. Aoyama Y, Yoshida Y, Sonoda Y. Deformylation of 32-oxo24,25-dihydrolanosterol by the purified cytochrome P45014DM (Lanosterol 14R-demethylase) from yeast evidence confirming the intermediate step of lanosterol 14Rdemethylation. J Biol Chem 1989;264:18502-5.

8. Chaudhary MP, G Tupe S, V Deshpande M. Chitin synthase inhibitors as antifungal agents. Mini Rev Med Chem 2013;13:222-36.

9. Sheng C, Zhang W, Zhang M. Homology modeling of lanosterol $14 \alpha$-demethylase of Candida albicans and Aspergillus fumigatus and insights into the enzyme-substrate interactions. J Biomol Struct Dyn 2004;22:91-9.

10. Guan Z, Chai X, Yu S. Synthesis, molecular docking, and biological evaluation of novel triazole derivatives as antifungal agents. Chem Biol Drug Des 2010;76:496-8.

11. Chai X, Zhang J, Cao Y. Design, synthesis and molecular docking studies of novel triazole as an antifungal agent. Eur J Med Chem 2011:46:3167-76.

12. Kelley LA, Mezulis S, Yates CM. The phyre2 web portal for protein modeling, prediction and analysis. Nature Protocols 2015;10:845-58.

13. Laskowski RA, MacArthur MW, Thornton JM. PROCHECK: validation of protein structure coordinates. International Tables of Crystallography, Vol. F. Crystallography of Biological Macromolecules. Kluwer Academic Publishers, The Netherlands; 2001. p. 722-5.

14. Kumar A, Bora U. Molecular docking studies of curcumin natura derivatives with DNA topoisomerase I and II-DNA complexes. Interdisciplinary Sci: Comput Life Sci 2014;6:285-91.

15. Morris GM, Goodsell DS, Halliday RS. Automated docking using a lamarckian genetic algorithm and an empirical binding free energy function. J Comput Chem 1998;19:1639-62.

16. Dupont S, Lemetais G, Ferreira T. Ergosterol biosynthesis: a fungal pathway for life on land? Evolution 2012;9:2961-8.

17. Maseet M, Khan N, Basir SF. Ergosterol biosynthesis and pathogenicity markers inhibition of candida albicans by fungus mediated silver nanoparticles. World J Pharm Pharm Sci 2016;2:600-18.
18. Prasad R, Shukla S, Singh A. Insights into candida lipids. In: Candida albicans: Cell Mol Biol; 2017. p. 417-28.

19. Chen SC, Sorrell TC. Antifungal agents. Med J Aust 2007;7:404.

20. Zhao J, Xu Y, Li C. Association of T916C (Y257H) mutation in candida albicans ERG11 with fluconazole resistance. Mycoses 2013;3:315-20.

21. Rajput SB, Karuppayil SM. Small molecules inhibit growth viability and ergosterol biosynthesis in candida albicans. Springer Plus 2013;2:26-32.

22. Ahmad A, Khan A, Akhtar F. Fungicidal activity of thymol and carvacrol by disrupting ergosterol biosynthesis and membrane integrity against candida. Eur J Clin Microbiol 2011;30:41-50.

23. Prasanna G, Ujwal A, Diliprajudominic S. A new pipeline to discover antimycotics by inhibiting ergosterol and riboflavin synthesis: the inspirations of siddha medicine. Med Chem Res 2014;23:2651-8.

24. Brown GD, Denning DW, Gow NA. Hidden killers: human fungal infections. Sci Transl Med 2012;4:1-9.

25. Cowen LE, Sanglard D, Howard SJ. Mechanisms of antifungal drug resistance. Cold Spring Harb Perspect Med 2014;5:1-22.

26. Morio F, Loge C, Besse B. Screening for amino acid substitutions in the Candida albicans Erg11 protein of azolesusceptible and azole-resistant clinical isolates: new substitutions and a review of the literature. Diagn Microbiol Infect Dis 2010;66:373-84.

27. Morschhauser J. The development of fluconazole resistance in Candida albicans-an example of microevolution of a fungal pathogen. J Microbiol 2016;54:192-10.

28. Mane A, Vidhate P, Kusro C. Molecular mechanisms associated with fluconazole resistance in clinical Candida albicans isolates from India. Mycoses 2016;59:93-7.

29. Marichal P, Koymans L, Willemsens S. Contribution of mutations in the cytochrome P450 14 $\alpha$-demethylase (Erg11p, Cyp51p) to azole resistance in Candida albicans. J Microbiol 1999;145:2701-13.

30. White TC, Marr KA, Bowden RA. Clinical, cellular, and molecular factors that contribute to antifungal drug resistance. Clin Microbiol Rev 1998;11:382-21.

31. Ruge E, Korting HC, Borelli C. Current state of threedimensional characterization of antifungal targets and its use for molecular modelling in drug design. Int J Antimicrob Agents 2005;26:427-41. 